\title{
A Weighted Runge-Kutta Discontinuous Galerkin Method for 3D Acoustic and Elastic Wave-Field Modeling
}

\author{
Xijun $\mathrm{He}^{1,4}$, Dinghui Yang ${ }^{2, *}$ and Xiao $\mathrm{Ma}^{3}$ \\ ${ }^{1}$ State Key Laboratory of Marine Resource Utilization in South China Sea, \\ Hainan University, Haikou 570228, P.R. China. \\ 2 Department of Mathematical Sciences, Tsinghua University, Beijing 100084, \\ P.R. China. \\ ${ }^{3}$ Department of Applied Mathematics, Northwestern Polytechnical University, \\ $X i^{\prime}$ an 710072 , P.R. China. \\ 4 Department of Mathematics, Colledge of Information Science and Technology, \\ Hainan University, Haikou 570228, P.R. China.
}

Received 24 March 2018; Accepted (in revised version) 8 October 2018

\begin{abstract}
Numerically solving 3D seismic wave equations is a key requirement for forward modeling and inversion. Here, we propose a weighted Runge-Kutta discontinuous Galerkin (WRKDG) method for 3D acoustic and elastic wave-field modeling. For this method, the second-order seismic wave equations in 3D heterogeneous anisotropic media are transformed into a first-order hyperbolic system, and then we use a discontinuous Galerkin (DG) solver based on numerical-flux formulations for spatial discretization. The time discretization is based on an implicit diagonal Runge-Kutta (RK) method and an explicit iterative technique, which avoids solving a large-scale system of linear equations. In the iterative process, we introduce a weighting factor. We investigate the numerical stability criteria of the 3D method in detail for linear and quadratic spatial basis functions. We also present a 3D analysis of numerical dispersion for the full discrete approximation of acoustic equation, which demonstrates that the WRKDG method can efficiently suppress numerical dispersion on coarse grids. Numerical results for several different 3D models including homogeneous and heterogeneous media with isotropic and anisotropic cases show that the 3D WRKDG method can effectively suppress numerical dispersion and provide accurate wave-field information on coarse mesh.
\end{abstract}

AMS subject classifications: 86-08, 86A15

Key words: Numerical modeling, anisotropy, discontinuous Galerkin method, numerical dispersion, stability.

*Corresponding author. Email addresses: hexijun111@sina.com (X. He), dhyang@math.tsinghua.edu.cn (D. Yang), maxiao@nwpu.edu.cn (X. Ma) 


\section{Introduction}

Accurate and efficient numerical method is necessary in computational geophysics for the purpose of resource explorations and understanding the earth. In recent years, there has been a strong interest for the discontinuous Galerkin method (DGM) in seismic numerical simulation. The DGM was first introduced in the early 1970s for solving the linear neutron transport equations [1], and later extended to solve hyperbolic conservation problems by employing discontinuous flux formulations and varies temporal discretizations. The work by Cockburn and Shu $[2,3]$ formulated the total variation diminishing (TVD) RK discontinuous Galerkin method into a complete mathematical framework. In computational seismology DGM has rapid developments [4-14]. For instance, an arbitrary high-order derivatives DGM proposed by Dumbser and Käser has been widely used in computational geophysics [6-11]. In 3D seismic modeling, the complex geologic geometry and large velocity contrast bring up many challenges to numerical methods due to the large amount of computations and storage requirements. However, the DGMs have been proved successfully to be efficient and high-order accurate in modeling seismic elastic wave propagating in complex media $[5,12]$, even with viscoelastic attenuation media [7].

The main difference between the DGM and traditional FEM is that the basis function can be discontinuous across elements in DGM. In general, DGM has the following advantages: (i) it maintains good properties with respect to conservation, stability, and convergence; (ii) it is easy to deal with domains with complex structures and non-conforming meshes, allowing for hanging nodes; (iii) the solution can be discontinuous across the element interfaces; (iv) complete localization means that DGM avoids dealing with large global mass matrices and is therefore especially suitable for parallel computing; (v) it is flexible for DGM to deal with locally varying polynomial degrees and element shapes (hp-adaptivity).

Many developed DGMs are based on the semi-discrete approach, wherein they employ the discontinuous Galerkin formulations for the spatial discretization, transforming the original partial differential equations (PDEs) into a system of ordinary differential equations (ODEs). After that, a time-stepping method is carried out to advance the solution in time, such as RK schemes [2], arbitrary high-order derivatives time stepping method [6] or the Lax-Wendroff method [15]. Explicit time-stepping scheme is widely used since it is easy to program. Alternatively, implicit solvers, such as diagonal implicitRK $[16,17]$ and Newton iterative methods $[18,19]$, are used in time discretization because they permit to use longer time steps. However, the shortcoming of fully implicit solvers is the extremely high computational cost induced by solving the large-scale linear algebraic equations. For this reason, explicit techniques such as the truncated differentiator series method and the predictor-corrector method have been developed (e.g., [20-22]), which turn to be robust methods to convert implicit methods into explicit ones and may preserve the good stability inherent in implicit schemes. In our research these techniques will be considered. 
Numerical dispersion in seismic modeling has been studied by many researchers as an important issue. Grid dispersion in the DGM has been analyzed by Hu et al. [23], using flux formulation DGM and Legendre basis functions to give a semi-discrete numerical dispersion relation for the scalar wave equation. Afterwards, Ainsworth et al. [24,25] carried out an analysis of the acoustic wave equation based on spatial discretization for $1 \mathrm{D}$ and 2D cases. De Basabe et al. [26] presented a comprehensive analysis of the 2D dispersion for high-order DGMs for acoustic and elastic wave equations based on leapfrog time discretization. Antonietti et al. [27] gave some detailed numerical dispersion results for 2D discontinuous Galerkin spectral elements method using simplicial meshes for elastic equations. Afterwards, Ferroni et al. [28] conducted a dispersion-dissipation analysis of 3D continuous and discontinuous spectral elements method on both tetrahedral and hexahedral elements, which gave the first 3D quantitative numerical dispersion analyses. To the best of our knowledge, there has not yet any quantitative dispersion results for 3D flux formulation DGMs. We will discuss and present our results in this work.

In this paper, we propose a weighted RKDG (WRKDG) method to solve 3D acoustic and elastic equations, which is actually a generalization for the 2D case [13]. The spatial discretization is carried out using the local Lax-Friedrichs numerical flux formulations, and an implicit diagonal RK method is employed to solve the semi-discrete ODEs. We employ a weighted two-step iterative process to make the implicit diagonal RK method explicit. As a result, the scheme is easy to implement and permits to use relatively longer time steps. The paper is organized as follows. In Section 2, we transform the secondorder 3D heterogeneous anisotropic wave equations into a first-order hyperbolic system. In Section 3, we derive the 3D WRKDG method in detail. In Section 4, detailed analyses of stability conditions and numerical dispersion relationship are deduced for the 3D acoustic equation. In the analyses, we consider regular hexahedral elements using orthogonal Legendre basis functions. Some numerical experiments based on regular hexahedrons are presented in Section 5. Section 6 summarizes the conclusions of the research.

\section{Transformation of the wave equations}

In a 3D heterogeneous elastic medium, the seismic wave equation can be written as:

$$
\left\{\begin{array}{l}
\rho \frac{\partial^{2} U}{\partial t^{2}}=\nabla \cdot \sigma+f, \\
\sigma=C: \varepsilon \\
\varepsilon=\frac{1}{2}\left[\nabla U+(\nabla U)^{T}\right],
\end{array}\right.
$$

where $\rho=\rho(x, y, z)$ is the density, $U=\left(u_{1}, u_{2}, u_{3}\right)^{T}$ is the displacement vector, $\sigma$ and $\varepsilon$ are the stress and strain tensors, respectively. $C=\left\{c_{i j k l}\right\}$ is the stiffness tensor, and $f=\left(f_{1}, f_{2}, f_{3}\right)^{T}$ is the external force-source vector. We can then write the stress-strain relation as follows 
by using the reduced Voigt notation:

$$
\left(\begin{array}{l}
\sigma_{11} \\
\sigma_{22} \\
\sigma_{33} \\
\sigma_{23} \\
\sigma_{13} \\
\sigma_{12}
\end{array}\right)=\left(\begin{array}{llllll}
c_{11} & c_{12} & c_{13} & c_{14} & c_{15} & c_{16} \\
c_{21} & c_{22} & c_{23} & c_{24} & c_{25} & c_{26} \\
c_{31} & c_{32} & c_{33} & c_{34} & c_{35} & c_{36} \\
c_{41} & c_{42} & c_{43} & c_{44} & c_{45} & c_{46} \\
c_{51} & c_{52} & c_{53} & c_{54} & c_{55} & c_{56} \\
c_{61} & c_{62} & c_{63} & c_{64} & c_{65} & c_{66}
\end{array}\right)\left(\begin{array}{c}
\varepsilon_{11} \\
\varepsilon_{22} \\
\varepsilon_{33} \\
2 \varepsilon_{23} \\
2 \varepsilon_{13} \\
2 \varepsilon_{12}
\end{array}\right),
$$

where $c_{i j}(x, y, z)$ are the elastic constants with $c_{i j}=c_{j i}$. There are 21 independent elastic constants in full anisotropic case. For an isotropic elastic medium, there are two independent constants $\lambda$ and $\mu$, known as the Lamé parameters, and the stress-strain relation (2.2) can be degraded as follows

$$
\left(\begin{array}{l}
\sigma_{11} \\
\sigma_{22} \\
\sigma_{33} \\
\sigma_{23} \\
\sigma_{13} \\
\sigma_{12}
\end{array}\right)=\left(\begin{array}{cccccc}
c_{11} & c_{12} & c_{12} & 0 & 0 & 0 \\
c_{12} & c_{11} & c_{12} & 0 & 0 & 0 \\
c_{12} & c_{12} & c_{11} & 0 & 0 & 0 \\
0 & 0 & 0 & c_{44} & 0 & 0 \\
0 & 0 & 0 & 0 & c_{44} & 0 \\
0 & 0 & 0 & 0 & 0 & c_{44}
\end{array}\right)\left(\begin{array}{c}
\varepsilon_{11} \\
\varepsilon_{22} \\
\varepsilon_{33} \\
2 \varepsilon_{23} \\
2 \varepsilon_{13} \\
2 \varepsilon_{12}
\end{array}\right),
$$

where $c_{11}=\lambda+2 \mu, c_{12}=\lambda, c_{44}=\mu$. For homogeneous vertically transversely-isotropic medium, $c_{11}, c_{12}, c_{13}, c_{33}, c_{44}$ are five independent elastic constants, $c_{66}=\left(c_{11}-c_{12}\right) / 2$, and Eq. (2.2) can be written as

$$
\left(\begin{array}{l}
\sigma_{11} \\
\sigma_{22} \\
\sigma_{33} \\
\sigma_{23} \\
\sigma_{13} \\
\sigma_{12}
\end{array}\right)=\left(\begin{array}{cccccc}
c_{11} & c_{12} & c_{13} & 0 & 0 & 0 \\
c_{12} & c_{11} & c_{13} & 0 & 0 & 0 \\
c_{13} & c_{13} & c_{33} & 0 & 0 & 0 \\
0 & 0 & 0 & c_{44} & 0 & 0 \\
0 & 0 & 0 & 0 & c_{44} & 0 \\
0 & 0 & 0 & 0 & 0 & c_{66}
\end{array}\right)\left(\begin{array}{c}
\varepsilon_{11} \\
\varepsilon_{22} \\
\varepsilon_{33} \\
2 \varepsilon_{23} \\
2 \varepsilon_{13} \\
2 \varepsilon_{12}
\end{array}\right) .
$$

We rewrite Eq. (2.1) in the following form

$$
\rho \frac{\partial^{2} U}{\partial t^{2}}=D \cdot U+f
$$

where $D$ is the second-order partial differential operator. For example, for the 3D coupled elastic anisotropic case, $D$ is defined by

$D=\frac{\partial}{\partial x}\left(C_{1} \frac{\partial}{\partial x}+C_{2} \frac{\partial}{\partial y}+C_{3} \frac{\partial}{\partial z}\right)+\frac{\partial}{\partial y}\left(C_{4} \frac{\partial}{\partial x}+C_{5} \frac{\partial}{\partial y}+C_{6} \frac{\partial}{\partial z}\right)+\frac{\partial}{\partial z}\left(C_{7} \frac{\partial}{\partial x}+C_{8} \frac{\partial}{\partial y}+C_{9} \frac{\partial}{\partial z}\right)$, where $C_{1}, C_{2}, C_{3}, C_{4}, C_{5}, C_{6}, C_{7}, C_{8}, C_{9}$ are parameter matrices related to the elastic constants $c_{i j}$. To transform Eq. (2.3) into a 1st-order linear hyperbolic system, we introduce three 
vectors $P=\left(p_{1}, p_{2}, p_{3}\right)^{T}, Q=\left(q_{1}, q_{2}, q_{3}\right)^{T}$ and $R=\left(r_{1}, r_{2}, r_{3}\right)^{T}$ such that $\frac{\partial P}{\partial t}=\frac{\partial U}{\partial x}, \frac{\partial Q}{\partial t}=\frac{\partial U}{\partial y}$, $\frac{\partial R}{\partial t}=\frac{\partial U}{\partial z}$. Using Eq. (2.3) and the definitions of $P, Q$ and $R$, Eq. (2.3) can be rewritten as:

$$
\left\{\begin{array}{l}
\rho \frac{\partial U}{\partial t}=\frac{\partial}{\partial x}\left(C_{1} P+C_{2} Q+C_{3} R\right)+\frac{\partial}{\partial y}\left(C_{4} P+C_{5} Q+C_{6} R\right)+\frac{\partial}{\partial z}\left(C_{7} P+C_{8} Q+C_{9} R\right)+\tilde{f} \\
\frac{\partial P}{\partial t}=\frac{\partial U}{\partial x} \\
\frac{\partial Q}{\partial t}=\frac{\partial U}{\partial y} \\
\frac{\partial R}{\partial t}=\frac{\partial U}{\partial z}
\end{array}\right.
$$

where $\tilde{f}$ denotes the integral of the external force-source $f$ with respect to $t$. Further, let

$$
\begin{aligned}
& W=\left(\begin{array}{c}
U \\
P \\
Q \\
R
\end{array}\right), \quad F(W)=-\left(\begin{array}{ccc}
\frac{C_{1}}{\rho} P+\frac{C_{2}}{\rho} Q+\frac{C_{3}}{\rho} R & \frac{C_{4}}{\rho} P+\frac{C_{5}}{\rho} Q+\frac{C_{6}}{\rho} R & \frac{C_{7}}{\rho} P+\frac{C_{8}}{\rho} Q+\frac{C_{9}}{\rho} R \\
U & 0 & 0 \\
0 & U & 0 \\
0 & 0 & U
\end{array}\right), \\
& \hat{f}=\left(\begin{array}{c}
\tilde{f} / \rho \\
0 \\
0 \\
0
\end{array}\right)
\end{aligned}
$$

then Eq. (2.4) has a simple form:

$$
\frac{\partial W}{\partial t}+\nabla \cdot F(W)=\hat{f}
$$

where $F(W)$ is the called the flux vector.

For the $3 \mathrm{D}$ acoustic equation

$$
u_{t t}-c^{2}\left(\frac{\partial^{2} u}{\partial x^{2}}+\frac{\partial^{2} u}{\partial y^{2}}+\frac{\partial^{2} u}{\partial z^{2}}\right)=f(t)
$$

Eq. (2.5) has the following concise form

$$
\left(\begin{array}{l}
u \\
p \\
q \\
r
\end{array}\right)_{t}+\nabla \cdot\left(\begin{array}{ccc}
-c^{2} p & -c^{2} q & -c^{2} r \\
-u & 0 & 0 \\
0 & -u & 0 \\
0 & 0 & -u
\end{array}\right)=\left(\begin{array}{l}
\tilde{f} \\
0 \\
0 \\
0
\end{array}\right)
$$

where

$$
W=\left(\begin{array}{c}
u \\
p \\
q \\
r
\end{array}\right), \quad F(W)=\left(\begin{array}{ccc}
-c^{2} p & -c^{2} q & -c^{2} r \\
-u & 0 & 0 \\
0 & -u & 0 \\
0 & 0 & -u
\end{array}\right), \quad \hat{f}=\left(\begin{array}{c}
\tilde{f} \\
0 \\
0 \\
0
\end{array}\right),
$$

and $c$ is the acoustic velocity. 


\section{Numerical scheme}

\subsection{Spatial discretization}

Using classical notations, the computational domain $\Omega \in \mathbb{R}^{3}$ is partitioned into nonoverlapping sub-elements $\left\{\Omega_{i}\right\}$. The scalar test function broken space of the DGM is

$$
V_{h}=\left\{v \in L^{1}(\Omega):\left.v\right|_{\Omega_{i}} \in P\left(\Omega_{i}\right)\right\}
$$

where $P\left(\Omega_{i}\right)$ is a polynomial space defined on $\Omega_{i}$. Let $v$ be a time-independent scalar test function, then an approximate solution $W$ of Eq. (2.6) satisfies

$$
\int_{\Omega_{i}}\left(v \frac{\partial W}{\partial t}+v \nabla \cdot F(W)\right) \mathrm{d} V=\int_{\Omega_{i}} \hat{f} v \mathrm{~d} V
$$

Using Green's formula, Eq. (3.1) is recast as

$$
\int_{\Omega_{i}}\left(v \frac{\partial W}{\partial t}-F(W) \cdot \nabla v\right) \mathrm{d} V+\int_{\partial \Omega_{i}} v F(W) \cdot n \mathrm{~d} S=\int_{\Omega_{i}} \hat{f} v \mathrm{~d} V
$$

where $\partial \Omega_{i}$ is the boundary of $\Omega_{i}$, and $n$ denotes the unit outward normal vector to $\partial \Omega_{i}$. Since $W$ may be discontinuous across the boundary $\partial \Omega_{i}$, we must replace the flux by a numerical flux $\hat{F}\left(W^{\text {int }}, W^{\text {ext }}, n\right)$, where $W^{\text {int }}$ and $W^{\text {ext }}$ are the traces of $W$ on $\partial \Omega_{i}$ taken from the interior and exterior of $\Omega_{i}$, respectively. By incorporating the Lipschitz, consistent and monotone numerical fluxes $[29,30]$, the DG schemes are known to be stable and convergent. Here, we use a standard local Lax-Friedrichs flux [2,3], which reads

$$
\hat{F}\left(W^{\mathrm{int}}, W^{\mathrm{ext}}, n\right)=\frac{1}{2}\left[F\left(W^{\mathrm{int}}\right)+F\left(W^{\mathrm{ext}}\right)\right] \cdot n-\frac{C_{\partial \Omega_{i}}}{2}\left(W^{\mathrm{ext}}-W^{\mathrm{int}}\right),
$$

where the numerical viscosity constant $C_{\partial \Omega_{i}}$ can be chosen to be the biggest eigenvalue (in absolute value) of $\frac{\partial}{\partial W} F\left(W^{\text {int }}\right) \cdot n$ and $\frac{\partial}{\partial W} F\left(W^{\text {ext }}\right) \cdot n$.

Let $\left\{w_{l}^{i}\right\}_{l=0}^{l=d_{l o c}-1}$ be a set of time-independent polynomial basis functions of $V_{h}$. Possible examples for $\left\{w_{l}^{i}\right\}_{l=0}^{l=d_{l o c}-1}$ include the Legendre polynomials for regular elements [2,3] or the simple monomial functions for triangular elements [31] or tetrahedral elements. The variable $d_{l o c}$ is the local dimension of the basis functions for typical 3D elements. In this study, we employ the 3D Legendre polynomials as basis functions, which is constructed by the tensor-product of 1D Legendre polynomials. The numerical solution of Eq. (3.2) can be written as the following numerical approximation:

$$
\left.W\right|_{\Omega_{i}}=\sum_{l=0}^{d_{l o c}-1} c_{l}^{i}(t) w_{l}^{i},
$$


in which $\left\{c_{l}^{i}(t)\right\}_{l=0}^{d_{l o c}-1}$ are coefficients, or called degrees of freedom (DOF). With Eq. (3.4), we replace $v$ with $w_{l^{\prime}}^{i}$ in Eq. (3.2), so that Eq. (3.2) reads as

$$
\begin{aligned}
& \sum_{l=0}^{d_{l o c}-1} \frac{\partial c_{l}^{i}(t)}{\partial t} \int_{\Omega_{i}} w_{l}^{i} w_{l^{\prime}}^{i} \mathrm{~d} V-\int_{\Omega_{i}} F\left(\sum_{l=0}^{d_{l o c}-1} c_{l}^{i}(t) w_{l}^{i}\right) \cdot\left(\frac{\partial w_{l^{\prime}}^{i}}{\partial x}, \frac{\partial w_{l^{\prime}}^{i}}{\partial y}, \frac{\partial w_{l^{\prime}}^{i}}{\partial z}\right) \mathrm{d} V \\
& \quad+\sum_{j} \int_{\Omega_{i} \cap \Omega_{j}} w_{l^{\prime}}^{i} \hat{F}\left(\left(\sum_{l=0}^{d_{l o c}-1} c_{l}^{i}(t) w_{l}^{i}\right),\left(\sum_{l=0}^{d_{l o c}-1} c_{l}^{j}(t) w_{l}^{j}\right), n\right) \mathrm{d} S=\int_{\Omega_{i}} \hat{f} w_{l^{\prime}}^{i} \mathrm{~d} V .
\end{aligned}
$$

\subsection{Time discretization}

As can be seen from Eq. (3.5), we are left to solve a semi-discrete ODE system. To simplify the expression, we introduce a coefficient vector $C^{i}(t)$ to represent the coefficients $\left\{c_{l}^{i}(t)\right\}_{l=0}^{d_{l o c}-1}$, and omit the superscript $i$ and the argument $t$. Then, the semi-discrete Eq. (3.5) reads

$$
\frac{\partial C}{\partial t}=L(C)+\bar{F}
$$

where $L$ is the linear operator with respect to $C . \bar{F}$ denotes the source excitation item.

The ODE system (3.6) can be advanced by means of an explicit treatment, e.g., the classic TVD RK time discretization [2,3]. To expect to improve the CFL stability conditions, following the techniques by He et al. [13], we employ the following implicit diagonal RK method to solve the semi-discrete system, which reads as

$$
C^{(n+1)}=C^{(n)}+\frac{\Delta t}{2}\left(K^{(n)}+\bar{K}^{(n)}\right),
$$

where

$$
\begin{aligned}
& K^{(n)}=L\left(C^{(n)}+r \Delta t K^{(n)}\right)+\bar{F}\left(t^{n}+r \Delta t\right), \\
& \bar{K}^{(n)}=L\left(C^{(n)}+(1-2 r) \Delta t K^{(n)}+r \Delta t \bar{K}^{(n)}\right)+\bar{F}\left(t^{n}+(1-r) \Delta t\right),
\end{aligned}
$$

where $r=(3-\sqrt{3}) / 6$.

In the above algorithm, we need to compute $K^{(n)}$ and $\bar{K}^{(n)}$ to obtain $C^{(n+1)}$ in Eq. (3.7). However, $K^{(n)}$ and $\bar{K}^{(n)}$ are provided implicitly, which means that we have to solve a set of linear algebraic equations to determine $K^{(n)}$ and $\bar{K}^{(n)}$ at each time advance. This will result in a great increase in computational cost. To avoid this, we try to convert the implicit scheme into an explicit one by means of iterative procedure. For this purpose, we first rewrite Eq. (3.8a) and ignore the source item, to obtain

$$
K^{(n)}=r \Delta t L\left(K^{(n)}\right)+L\left(C^{(n)}\right) .
$$

If $\|r \Delta t L\|<1$, then Eq. (3.9) implies that the following iterative process can be used:

$$
K_{k+1}^{(n)}=r \Delta t L\left(K_{k}^{(n)}\right)+L\left(C^{(n)}\right),
$$


where the subscript $k$ is the iterative number. Always, we can implement more iterations to achieve higher time accuracy. However, when taking the amount of computation into account, we favor the use of a few steps of iterations to save computational costs. We choose $L\left(C^{(n)}\right)$ as the initial value of $K_{0}^{(n)}$, and apply two iterations, then we obtain the following scheme:

$$
\left\{\begin{array}{l}
K_{0}^{(n)}=L\left(C^{(n)}\right) \\
K_{1}^{(n)}=r \Delta t L\left(K_{0}^{(n)}\right)+L\left(C^{(n)}\right) \\
K_{2}^{(n)}=r \Delta t L\left(K_{1}^{(n)}\right)+L\left(C^{(n)}\right)
\end{array}\right.
$$

$K_{1}^{(n)}$ and $K_{2}^{(n)}$ can be suitable approximations of $K^{(n)}$. For simplicity, we can take $K_{2}^{(n)}$ as an approximation of $K^{(n)}$. However, to enrich the numerical method, a better alternative is to take an intermediate value between $K_{2}^{(n)}$ and $K_{1}^{(n)}$ for the approximation of $K^{(n)}$, which reads

$$
\begin{aligned}
K^{(n)} & =\eta K_{2}^{(n)}+(1-\eta) K_{1}^{(n)} \\
& =L\left(C^{(n)}\right)+r \Delta t L^{2}\left(C^{(n)}\right)+\eta(r \Delta t)^{2} L^{3}\left(C^{(n)}\right),
\end{aligned}
$$

where $\eta \in[0,1]$. The above stepping scheme is of second order accuracy in time discretization, unless $\eta=1$ gives third order of accuracy. To compute $\bar{K}^{(n)}$ in Eq. (3.8b), we define $T^{(n)}=C^{(n)}+(1-2 r) \Delta t K^{(n)}$. If we ignore the source term, Eq. (3.8b) can be rewritten as:

$$
\bar{K}^{(n)}=r \Delta t L\left(\bar{K}^{(n)}\right)+L\left(T^{(n)}\right) .
$$

We can implement the same process as (3.10) to obtain $\bar{K}^{(n)}$, if we take $L\left(T^{(n)}\right)$ as the iterative initial value for $\bar{K}^{(n)}$.

\section{Stability and numerical dispersion}

\subsection{Von Neumann analysis}

We conduct a Von Neumann analysis in order to derive the numerical stability conditions and dispersion errors of the 3D WRKDG. We focus on the 3D acoustic wave equation in an isotropic medium and further suppose that the medium is unbounded and sourcefree. These hypotheses have been widely used [23-28]. We recall that the flux $F(W)$ is linear, then we can rewrite the flux vector in Eq. (2.7) in the following format [23]:

$$
F(W)=\left(A_{1} W, A_{2} W, A_{3} W\right)
$$

where

$$
A_{1}=\left(\begin{array}{cccc}
0 & -c^{2} & 0 & 0 \\
-1 & 0 & 0 & 0 \\
0 & 0 & 0 & 0 \\
0 & 0 & 0 & 0
\end{array}\right), \quad A_{2}=\left(\begin{array}{cccc}
0 & 0 & -c^{2} & 0 \\
0 & 0 & 0 & 0 \\
-1 & 0 & 0 & 0 \\
0 & 0 & 0 & 0
\end{array}\right), \quad A_{3}=\left(\begin{array}{cccc}
0 & 0 & 0 & -c^{2} \\
0 & 0 & 0 & 0 \\
0 & 0 & 0 & 0 \\
-1 & 0 & 0 & 0
\end{array}\right) \text {. }
$$


After some algebraic operations, the numerical flux $\hat{F}\left(W^{\text {int }}, W^{\text {ext }}, n\right)$ in Eq. (3.3) can be written in the following form:

$$
\hat{F}\left(W^{\text {int }}, W^{\text {ext }}, n\right)=\hat{A}^{\text {int }} W^{\text {int }}+\hat{A}^{\text {ext }} W^{\text {ext }},
$$

where

$$
\begin{aligned}
& \hat{A}^{\mathrm{int}}=\frac{1}{2}\left(A_{1} n_{1}+A_{2} n_{2}+A_{3} n_{3}+C_{\partial \Omega_{i}} I\right), \\
& \hat{A}^{\mathrm{ext}}=\frac{1}{2}\left(A_{1} n_{1}+A_{2} n_{2}+A_{3} n_{3}-C_{\partial \Omega_{i}} I\right), \\
& n=\left(n_{1}, n_{2}, n_{3}\right)^{T},
\end{aligned}
$$

where $I$ is the identity matrix.

Next, the domain is partitioned into regular hexahedral elements $E^{n, m, k}=\left[x_{n}, x_{n+1}\right] \times$ $\left[y_{m}, y_{m+1}\right] \times\left[z_{k}, z_{k+1}\right]$, with sides parallel to the coordinate axes with $\delta_{x}=x_{n+1}-x_{n}, \delta_{y}=$ $y_{m+1}-y_{m}$ and $\delta_{z}=z_{k+1}-z_{k}$. In order to facilitate the analysis, it is usually assumed that $\delta_{x}=\delta_{y}=\delta_{z}=h$. The approximation of $W$ inside $E^{n, m, k}$ can be written as

$$
\left.W\right|_{E^{n, m, k}}=\sum_{l=0}^{d_{l o c}-1} C_{l}^{n, m, k}(t) w_{l}^{n, m, k}
$$

where $C_{l}^{n, m, k}(t)$ are DOF for the solution, and $w_{l}^{n, m, k}$ are the local basis set in the element $E^{n, m, k}(t)$. According to the numerical flux in Eqs. (4.1) and (4.2), Eq. (3.5) can be rewritten as

$$
\begin{aligned}
& \sum_{l=0}^{d_{l o c}-1}\{ \frac{\partial C_{l}^{n, m, k}}{\partial t} \int_{E^{n, m, k}} w_{l}^{n, m, k} w_{l^{\prime}}^{n, m, k} \mathrm{~d} V-\sum_{k=1}^{3} A_{k} C_{l}^{n, m, k} \int_{E^{n, m, k}} w_{l}^{n, m, k} \frac{\partial w_{l^{\prime}}^{n, m, k}}{\partial x_{k}} \mathrm{~d} V \\
&\left.+\sum_{i=1}^{6} \hat{A}^{\mathrm{int}} C_{l}^{n, m, k} \int_{F_{i}} w_{l}^{n, m, k} w_{l^{\prime}}^{n, m, k} \mathrm{~d} S+\sum_{i=1}^{6} \hat{A}^{\mathrm{ext}} C_{l}^{n^{\prime}, m^{\prime}, k^{\prime}} \int_{F_{i}} w_{l}^{n^{\prime}, m^{\prime} k^{\prime}} w_{l^{\prime}}^{n, m, k} \mathrm{~d} S\right\}=0 \\
& l^{\prime}=0, \cdots, d_{l o c}-1
\end{aligned}
$$

where $F_{i}(i=1, \cdots, 6)$ denotes the six faces of $E^{n, m, k}$.

Let $C^{n, m, k}(t)=\left(C_{0}^{n, m, k}, C_{1}^{n, m, k}, \cdots, C_{d_{l o c}-m}^{n, m}\right)^{T}$, then Eq. (4.3) can be written as:

$$
\begin{aligned}
& Q \frac{\partial C^{n, m, k}}{\partial t}+\frac{2}{\delta_{x}}\left[N_{0} C^{n, m, k}+N_{-1} C^{n-1, m, k}+N_{+1} C^{n+1, m, k}\right]+\frac{2}{\delta_{y}}\left[M_{0} C^{n, m, k}\right. \\
& \left.\quad+M_{-1} C^{n, m-1, k}+M_{+1} C^{n, m+1, k}\right]+\frac{2}{\delta_{z}}\left[K_{0} C^{n, m, k}+K_{-1} C^{n, m, k-1}+K_{+1} C^{n, m, k+1}\right]=0,
\end{aligned}
$$

wherein the explicit forms of these matrices are shown in Appendix A. 
We choose the following plane-wave solution

$$
C^{n, m, k}(t)=C(t) \exp \left[i\left(\kappa \sin \theta \cos \varphi n \delta_{x}+\kappa \sin \theta \sin \varphi m \delta_{y}+\kappa \cos \theta k \delta_{z}\right)\right],
$$

which represents a plane wave with wave number $\kappa$ and propagation direction determined by $\theta$ and $\varphi . \theta \in[0, \pi]$ denotes the angle between the propagation direction and the $z$-axis, and $\varphi \in[0,2 \pi]$ denotes the angle between the projection of the propagation direction into the $x-y$ plane and $x$-axis. By substituting the wave expression into Eq. (4.4), we get

$$
\begin{aligned}
\frac{\partial C(t)}{\partial t}=-Q^{-1}[ & \frac{2}{h}\left(N_{0}+N_{-1} e^{-i \kappa \sin \theta \cos \varphi h}+N_{+1} e^{i \kappa \sin \theta \cos \varphi h}\right)+\frac{2}{h}\left(M_{0}+M_{-1} e^{-i \kappa \sin \theta \sin \varphi h}\right. \\
& \left.\left.+M_{+1} e^{i \kappa \sin \theta \sin \varphi h}\right)+\frac{2}{h}\left(K_{0}+K_{-1} e^{-i \kappa \cos \theta h}+K_{+1} e^{i \kappa \cos \theta h}\right)\right] C(t) .
\end{aligned}
$$

We introduce a variable $S$ to simplify the RHS of Eq. (4.5), which is now written as

$$
\frac{\partial C(t)}{\partial t}=S C(t)
$$

where

$$
\begin{aligned}
S= & -Q^{-1}\left[\frac{2}{h}\left(N_{0}+N_{-1} e^{-i \kappa \sin \theta \cos \varphi h}+N_{+1} e^{i \kappa \sin \theta \cos \varphi h}\right)\right. \\
& \left.+\frac{2}{h}\left(M_{0}+M_{-1} e^{-i \kappa \sin \theta \sin \varphi h}+M_{+1} e^{i \kappa \sin \theta \sin \varphi h}\right)+\frac{2}{h}\left(K_{0}+K_{-1} e^{-i \kappa \cos \theta h}+K_{+1} e^{i \kappa \cos \theta h}\right)\right] .
\end{aligned}
$$

In order to solve Eq. (4.6), we use the weighted RK time discretization method introduced in Subsection 3.2. After some algebraic operations, we obtain

$$
C^{n+1}=\left(I+\frac{\Delta t}{2}\left(G_{1}+G_{1} G_{2}\right)\right) C^{n}
$$

where

$$
\begin{aligned}
& G_{1}=S+r \Delta t S^{2}+\eta(r \Delta t)^{2} S^{3}, \\
& G_{2}=I+(1-2 r) \Delta t G_{1} .
\end{aligned}
$$

Next, we assume that $C^{n}=C^{0} e^{-i \omega n \Delta t}$, where $\omega$ is the numerical angular frequency, and $C^{0}$ is an arbitrary constant vector. Then Eq. (4.7) can be written as

$$
e^{-i \omega \Delta t} C^{0}=\left(I+\frac{\Delta t}{2}\left(G_{1}+G_{1} G_{2}\right)\right) C^{0} .
$$

Let $\Lambda$ denote the eigenvalue of the matrix on the RHS of Eq. (4.8), i.e.,

$$
e^{-i \omega \Delta t}=\Lambda \text {. }
$$

We remark that $\Lambda$ and $\omega$ are complex numbers. Eqs. (4.7)-(4.9) imply the stability and dispersion relationship. $\Lambda$ relates to the numerical stability condition, and $\omega$ leads to the numerical dispersion relationship. 


\subsection{Stability conditions}

We now consider the stability conditions of the WRKDG based on the above plane wave analysis. Note that the value of $\Lambda$ relates to the wave number $\kappa h$, propagation direction $\theta$ and $\varphi$, Courant number $\alpha$, and the weight $\eta$. From Eq. (4.7) and the definition of $\Lambda$, we know that to keep the scheme stable, $\Lambda$ must satisfy $|\Lambda| \leq 1$. Moreover, the modulus of all the eigenvalues of Eq. (4.8) must be no more than 1 for all the wave numbers $\kappa h \in[0, \pi]$ and all the propagation directions $\theta \in[0, \pi]$ and $\varphi \in[0,2 \pi]$, which can be described by an optimization problem:

$\max \alpha$

s.t. $|\Lambda(\kappa h, \alpha, \theta, \varphi)| \leq 1$ for $\kappa h \in[0, \pi], \theta \in[0, \pi]$ and $\varphi \in[0,2 \pi]$,

$\alpha \geq 0$

The solution for this problem can be numerically obtained. We resolve it for the linear and quadratic regular hexahedral cases, whose spatial accuracy is 2nd- and 3rd-order, respectively. Fig. 1 plots the different maximal Courant numbers for different weights $\eta$ from 0 to 1 . If we denote a spatial interpolation of the polynomial of degree at most $N$ by $P^{N}$, then for the $P^{1}$ case, the maximal Courant number $\alpha_{\max }$ varies from 0.4 to 0.888 , as the weight $\eta$ increases from 0 to 1 . For example, the stability condition with $\eta=0.36$ is given by

$$
\Delta t \leq \alpha_{\max } \frac{h}{c} \approx 0.888 \frac{h}{c} .
$$

For the $P^{2}$ case, the maximal Courant number $\alpha_{\max }$ varies from 0.217 to 0.310 , as the weight $\eta$ increases from 0 to 1 . For example, the stability condition with $\eta=0.56$ is given by

$$
\Delta t \leq \alpha_{\max } \frac{h}{c} \approx 0.310 \frac{h}{c} .
$$

For comparison purpose, we also give the detailed stability conditions of the 3rdorder DGM with the 3rd-order TVD RK time discretization for the homogeneous acoustic equation. For the $P^{1}$ case, the stability condition is given by

$$
\Delta t \leq \alpha_{\max } \frac{h}{c} \approx 0.260 \frac{h}{c},
$$

and for the $P^{2}$ case, the stability condition is given by

$$
\Delta t \leq \alpha_{\max } \frac{h}{c} \approx 0.136 \frac{h}{c} .
$$

Comparing the stability conditions in Eqs. (4.10)-(4.13) for the $P^{1}$ and $P^{2}$ elements, respectively, we can see that the stability conditions of the WRKDG methods are more relaxed than those of the 3rd-order DGM with the TVD RK time discretization. In particular, there is a about 3.4-fold difference in the maximal Courant number for $P^{1}$ element 


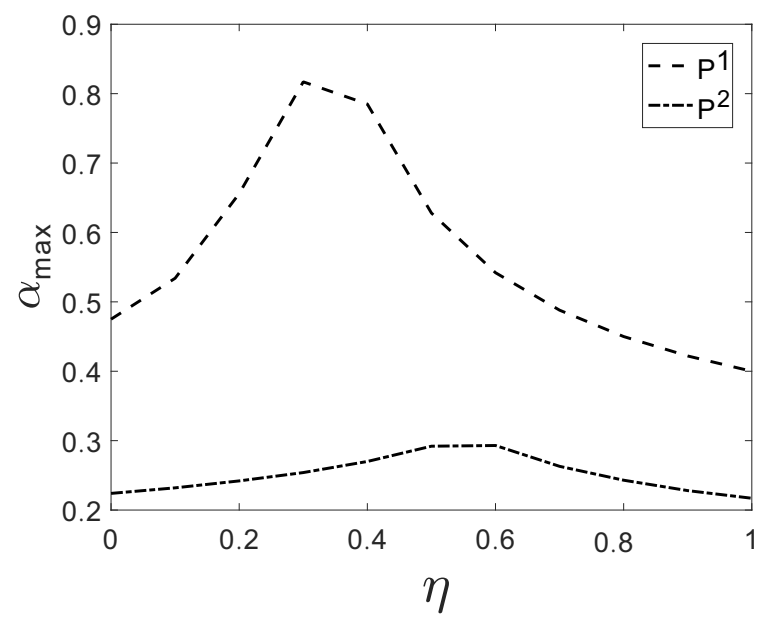

Figure 1: The maximal Courant numbers of the 3D WRKDG method for $P^{1}$ and $P^{2}$ elements for weighting factors $\eta$ varying from 0 to 1 .

and a 2.3-fold difference for $P^{2}$ element between the two numerical methods. The results is comparable with the 2D WRKDG method in [13]. It should be noted that the computational cost for each time-marching is 2 times larger than the TVD RK time discretization, hence the weighted RK time stepping scheme still achieve a 1.7 times superiority for $P^{1}$ element and is comparable to the TVD RK time discretization for $P^{2}$ element in computational efficiency.

Note that the stability conditions for heterogeneous media cannot be directly determined but could be approximated using local homogeneous cases. Our conjecture is that Eqs. (4.10) and (4.11) are approximately correct for heterogeneous media if the maximal wave velocity $c$ is used. The basis functions used here is order-complete Legendre basis. If we use other nonorthogonal basis, the above analysis approach is still applicable.

\subsection{Dispersion analysis}

It is well known that numerical dispersion is a major artifact when numerical methods are used to model wave fields. In this subsection, we investigate the numerical dispersion of the 3D WRKDG by using the above plane wave analysis, including the effects of the space and time discretization. We have already stated that $\Lambda$ and $\omega$ in Eqs. (4.8) and (4.9) are usually complex numbers. We therefore write $\omega=\omega_{r}+i \omega_{i}$ and $\Lambda=\Lambda_{r}+i \Lambda_{i}$, in which $\Lambda_{r}$ and $\Lambda_{i}$ are the real and imaginary parts of $\Lambda . \omega_{r}$, the real part of $\omega$, is related to numerical dispersion, while $\omega_{i}$ (the imaginary part) is related to the numerical damping inherent in the discretization process. A stable numerical scheme always has a non-positive $\omega_{i}$ value, which represents that the energy does not increase. Substituting the decomposition of $\Lambda$ and $\omega$ into Eq. (4.9), we have

$$
\Lambda_{r}+i \Lambda_{i}=e^{-i\left(\omega_{r}+i \omega_{i}\right) \Delta t}=e^{\omega_{i} \Delta t}\left(e^{-i \omega_{r} \Delta t}\right)=e^{\omega_{i} \Delta t}\left(\cos \left(\omega_{r} \Delta t\right)-i \sin \left(\omega_{r} \Delta t\right)\right) .
$$


Solving for $\omega_{r} \Delta t$, we then have

$$
\omega_{r} \Delta t=\arctan \left(-\frac{\Lambda_{i}}{\Lambda_{r}}\right)
$$

The numerical dispersion $R$ is defined by the ratio of numerical velocity $c_{\text {num }}$ and physical velocity $c$ :

$$
R=\frac{c_{\mathrm{num}}}{c}=\frac{\omega_{r}}{c \kappa}=\frac{\omega_{r} \Delta t}{\alpha \kappa h},
$$

where $\alpha=c \Delta t / h$ is the Courant number. If we adopt the definition of the spatial sampling ratio proposed by Moczo et al. [32], $S_{p}=\kappa h /(2 \pi)$, then we get

$$
R=\frac{\omega_{r} \Delta t}{2 \pi \alpha S_{p}}
$$

Figs. 2 and 3 shows how the numerical dispersion $R$ varies with sampling ratio $S_{p} \in$ $[0,0.5]$ for propagation directions $\theta=\pi / 2$ and $\theta=\pi / 4$, respectively, when $\alpha=0.29$. We give the results for four cases $\varphi=0^{\circ}, 15^{\circ}, 30^{\circ}, 45^{\circ}$. Figures (a) and (b) shown in Figs. 2
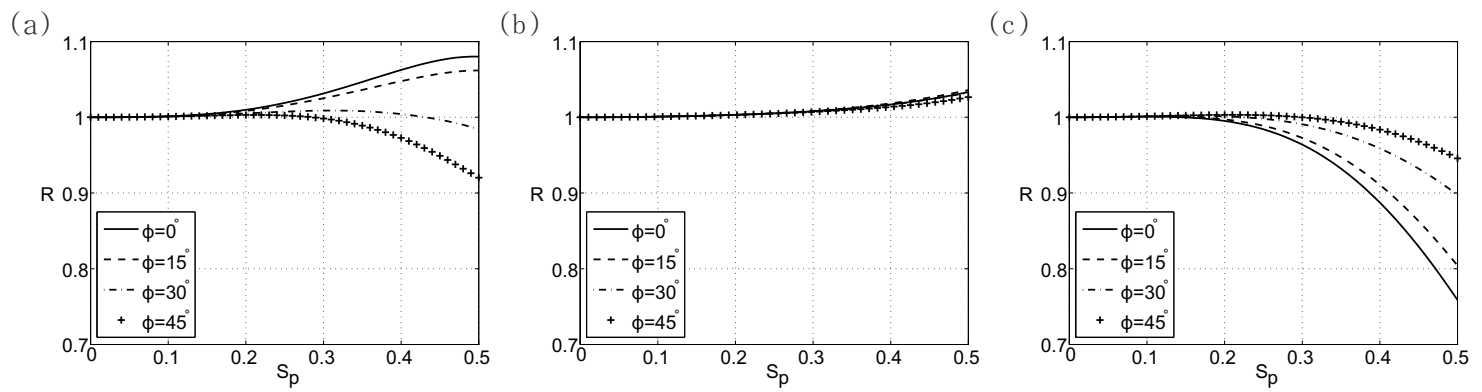

Figure 2: Numerical dispersion $R$ as a function of sampling rate $S_{p}$ for the different methods when $\theta=\pi / 2$ and $\varphi=0^{\circ}, 15^{\circ}, 30^{\circ}, 45^{\circ}$ : (a) WRKDG method for $P^{1}$ element, (b) WRKDG method for $P^{2}$ element, and (c) the 4th-order SG method.
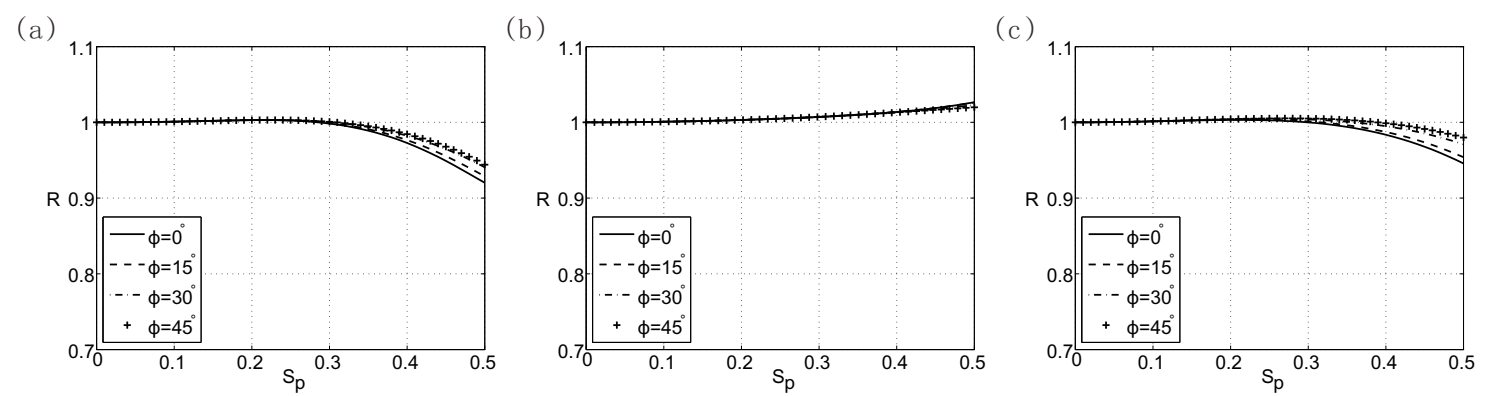

Figure 3: Numerical dispersion $R$ as a function of sampling rate $S_{p}$ for the different methods when $\theta=\pi / 4$ and $\varphi=0^{\circ}, 15^{\circ}, 30^{\circ}, 45^{\circ}$ : (a) WRKDG method for $P^{1}$ element, (b) WRKDG method for $P^{2}$ element, and (c) the 4th-order SG method. 

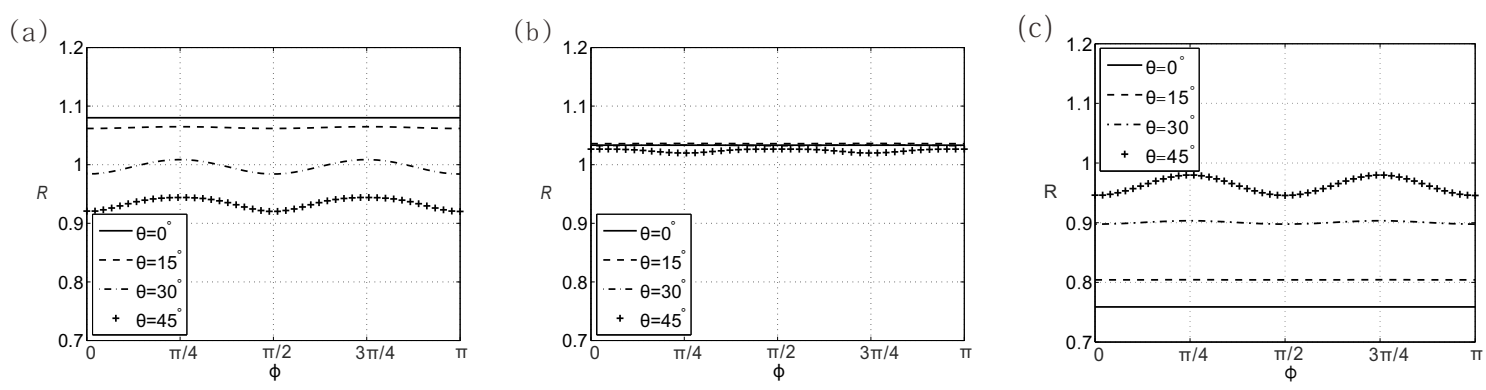

Figure 4: Numerical dispersion $R$ as a function of propagation direction $\varphi$ for the different methods when $S_{p}=0.5$ and $\theta=0^{\circ}, 15^{\circ}, 30^{\circ}, 45^{\circ}$ : (a) WRKDG method for $P^{1}$ element, (b) WRKDG method for $P^{2}$ element, and (c) the 4th-order SG method.

and 3 are the values using the $P^{1}$ and $P^{2}$ WRKDG method with $\eta=0.5$, respectively, whereas Fig. 2(c) and Fig. 3(c) illustrate the values for the 4th-order staggered-grid (SG) method [32] for comparison. We can see that as $S_{p}$ increases, the curves for the three methods deviate from $R=1$, which indicates that coarser sampling gives larger numerical dispersion. The two figures also suggest that the numerical dispersive error with $\theta=\pi / 4$ is much less than $\theta=\pi / 2$. In the three methods, the $P^{2}$ WRKDG method produces the smallest numerical dispersion, while the $P^{1}$ WRKDG method and the SG method are almost equivalent. We also observe that for the $P^{2}$ WRKDG method and SG method, as $\varphi$ increase, the numerical dispersion decreases, while the deviation is minimal at $\theta=\pi / 4$. In addition, for the $P^{2}$ WRKDG method, the numerical dispersion $R>1$, which means the waves are slightly advanced; for the SG method, the numerical dispersion $R<1$, which means the waves are slightly delayed; while for the $P^{1}$ WRKDG method, the numerical dispersion oscillates around $R=1$, which implies that the waves are delayed or advanced in different propagation directions. From Figs. 2 and 3 we can observe that the numerical dispersion is related to the propagation direction. In other words, numerical dispersion is anisotropic. In order to have some insights into the numerical dispersion anisotropy, we give some results about the influence of $\theta$ and $\varphi$ at $S_{p}=0.5$. Fig. 4 shows how the numerical dispersion $R$ varies for different propagation angles $\varphi \in[0, \pi]$ for $\theta=0^{\circ}, 15^{\circ}, 30^{\circ}, 45^{\circ}$. We can see that the anisotropy curves for the three methods are symmetric about $\varphi=\pi / 2$, while the numerical dispersion reaches its extreme points at $\theta=\pi / 4$ and $\theta=\pi / 2$. Fig. 5 shows how the numerical dispersion $R$ varies for different propagation angles $\theta \in[0, \pi]$ for $\varphi=0^{\circ}, 15^{\circ}, 30^{\circ}, 45^{\circ}$. We can see that for the $P^{2}$ WRKDG method and SG method, as $\varphi$ increase from 0 to $45^{\circ}$, the numerical dispersion decreases, while the deviation is minimal at $\varphi=\pi / 4$, which is consistent with the results in Figs. 2 and 3. Figs. 4 and 5 also suggest that the $P^{1}$ WRKDG method and the SG method have greater numerical dispersion anisotropy than the $P^{2}$ WRKDG method.

For further comparisons, we give the maximum numerical dispersive error $|1-R|$ for these methods for different Courant numbers, for different weighting factors, for $S_{p} \in[0,0.5], \theta \in[0, \pi], \varphi \in[0,2 \pi]$ in Table 1 . A blank entry means that the numerical 

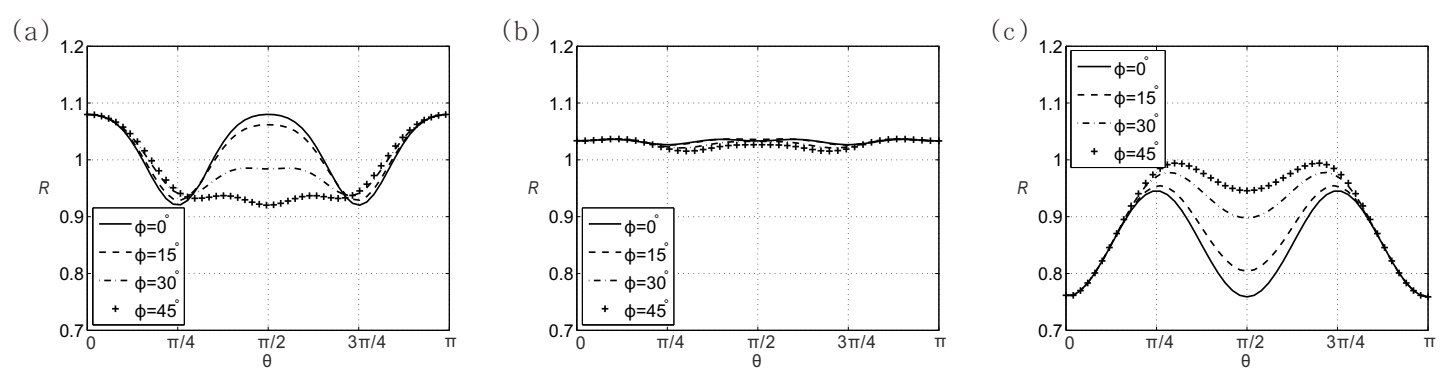

Figure 5: Numerical dispersion $R$ as a function of propagation direction $\theta$ for the different methods when $S_{p}=0.5$ and $\varphi=0^{\circ}, 15^{\circ}, 30^{\circ}, 45^{\circ}$ : (a) WRKDG method for $P^{1}$ element, (b) WRKDG method for $P^{2}$ element, and (c) the 4th-order SG method.

method is unstable for that specific Courant number. We can see from the table, for example, when $\alpha=0.16$, the maximum numerical dispersion errors for the $P^{1}$ and $P^{2}$ WRKDG method with $\eta=0.5$ are $8.883 \%$ and $2.182 \%$, respectively, while that of the SG method is $25.290 \%$. Table 1 demonstrates that the WRKDG method can effectively suppress numerical dispersion than SG method. By comparing with the numerical dispersion results in [13], we conclude that the numerical dispersive error has only a little difference between 3-D and 2-D cases.

Table 1: The maximum numerical dispersive errors for different Courant numbers for $S_{p} \in[0,0.5], \theta \in[0, \pi]$ and $\varphi \in[0,2 \pi](\%)$.

\begin{tabular}{||c|c|c|c|c|c|c|c||}
\hline \multirow{2}{*}{$\alpha$} & \multicolumn{3}{|c|}{ WRKDG $P^{1}$} & \multicolumn{3}{c||}{ WRKDG $P^{2}$} & \multirow{2}{*}{ SG } \\
\cline { 2 - 8 } & $\eta=0.0$ & $\eta=0.5$ & $\eta=1.0$ & $\eta=0.0$ & $\eta=0.5$ & $\eta=1.0$ & \\
\hline 0.1 & 8.844 & 9.038 & 9.030 & 2.051 & 1.817 & 1.582 & 25.558 \\
\hline 0.16 & 8.487 & 8.883 & 9.060 & 2.787 & 2.182 & 1.577 & 25.290 \\
\hline 0.29 & 10.487 & 7.964 & 9.286 & - & 3.543 & - & 24.232 \\
\hline 0.5 & - & 13.746 & - & - & - & - & 20.699 \\
\hline
\end{tabular}

\section{Numerical simulations}

In this section, we present some numerical examples to further illustrate the accuracy and capability of the 3D WRKDG method. Overall, considering the computational cost and accuracy, we use the $P^{2}$ WRKDG method for the numerical modeling. Since the amount of the computation and storage is huge for 3D models, stand-alone work has been unable to meet the needs. All the numerical tests are implemented with Message Passing Interface (MPI) parallel programming technology. 


\subsection{Convergence test}

To investigate the convergence rate of the WRKDG method, we consider the 3D homogeneous acoustic wave equation in Eq. (2.7), with the following exact solution

$$
\left\{\begin{array}{l}
u(t, x, y, z)=\cos \left(2 \pi f_{0} t-\frac{2 \pi f_{0}}{c} \sin \theta_{0} \cos \varphi_{0} x-\frac{2 \pi f_{0}}{c} \sin \theta_{0} \sin \varphi_{0} x-\frac{2 \pi f_{0}}{c} \cos \theta_{0} z\right), \\
p(t, x, y, z)=-\frac{\sin \theta_{0} \cos \varphi_{0}}{c} \cos \left(2 \pi f_{0} t-\frac{2 \pi f_{0}}{c} \sin \theta_{0} \cos \varphi_{0} x-\frac{2 \pi f_{0}}{c} \sin \theta_{0} \sin \varphi_{0} x-\frac{2 \pi f_{0}}{c} \cos \theta_{0} z\right), \\
q(t, x, y, z)=-\frac{\sin \theta_{0} \sin \varphi_{0}}{c} \cos \left(2 \pi f_{0} t-\frac{2 \pi f_{0}}{c} \sin \theta_{0} \cos \varphi_{0} x-\frac{2 \pi f_{0}}{c} \sin \theta_{0} \sin \varphi_{0} x-\frac{2 \pi f_{0}}{c} \cos \theta_{0} z\right), \\
r(t, x, y, z)=-\frac{\cos \theta_{0}}{c} \cos \left(2 \pi f_{0} t-\frac{2 \pi f_{0}}{c} \sin \theta_{0} \cos \varphi_{0} x-\frac{2 \pi f_{0}}{c} \sin \theta_{0} \sin \varphi_{0} x-\frac{2 \pi f_{0}}{c} \cos \theta_{0} z\right),
\end{array}\right.
$$

where $c$ is the acoustic velocity, $\theta_{0}$ and $\varphi_{0}$ denotes the incident direction at time $t=0$, and $f_{0}$ is the frequency. We choose the solution in Eq. (5.1) with time $t=0$ as the initial condition. The computational region is $0 \leq x, y, z \leq 1.8 \mathrm{~km}$ and the time step size is set to be $\Delta t=0.1 \mathrm{~ms}$, such that the time discretization errors can be ignored. We choose the parameters $c=4 \mathrm{~km} / \mathrm{s}, f_{0}=10 \mathrm{~Hz}, \theta_{0}=\pi / 4, \varphi_{0}=\pi / 4$, and perform the numerical experiment on regular hexahedral mesh. We run 3000 steps to study the numerical errors of the WRKDG method. We use the $P^{2}$ WRKDG method with a weighting factor of $\eta=0.5$ in the numerical experiment. In our numerical test, we find that the value of the weight $\eta$ has little effect on the numerical errors.

In order to investigate the convergence of this method, we define the continuous $L^{2}$ norm error between the numerical solution $u_{h}$ and the exact solution $u$ :

$$
E_{L^{2}}=\left\|u_{h}-u\right\|_{L^{2}}=\left(\int_{\Omega}\left|u_{h}-u\right|^{2} d \Omega\right)^{\frac{1}{2}} .
$$

If we choose two different mesh spacing $h^{s}$ and $h^{s-1}$ for the computational domain, then the convergence orders are computed through

$$
O_{L^{2}}=\log \left(\frac{E_{L^{2}}^{s}}{E_{L^{2}}^{s-1}}\right) / \log \left(\frac{h^{s}}{h^{s-1}}\right) .
$$

Fig. 6 shows the numerical error and the convergence result for the displacement $u$. We test for two cases: $f_{0}=20 \mathrm{~Hz}$ and $f_{0}=10 \mathrm{~Hz}$. We observe that with the decrease of the spatial increment $h$, the numerical error decreases, which implies the proposed method is convergent. In particular, we can see that the convergence order is about 3.

\subsection{Homogeneous isotropic acoustic model}

In this example, we employ the 3D WRKDG method to simulate the wave propagation for the homogeneous medium. The velocity is $4 \mathrm{~km} / \mathrm{s}$. The computational region is $0 \leq x, y, z \leq 11 \mathrm{~km}$. The explosive source in Eq. (2.7) is a Ricker wavelet

$$
f(t)=-9.6 f_{0}\left(0.6 f_{0} t-1\right) \times \exp \left[-8\left(0.6 f_{0} t-1\right)^{2}\right],
$$




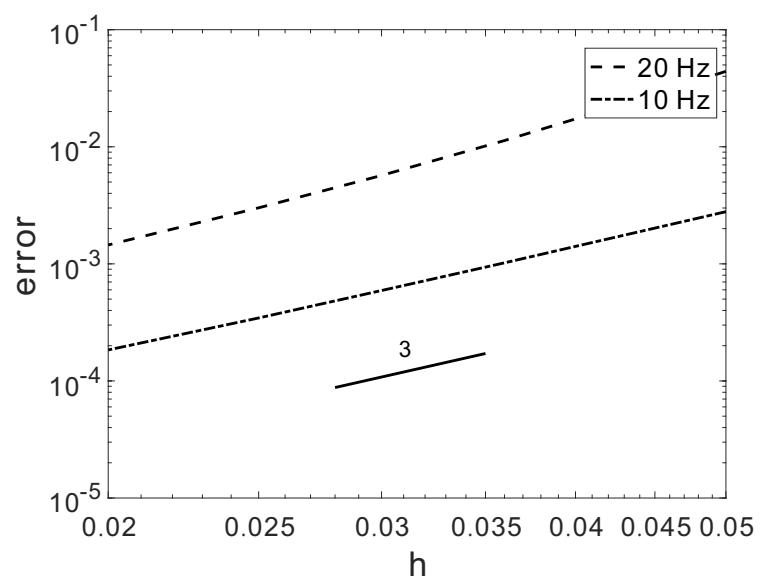

Figure 6: Numerical errors versus mesh size for $f_{0}=20 \mathrm{~Hz}$ and $f_{0}=10 \mathrm{~Hz}$.

with frequency $f_{0}=20 \mathrm{~Hz}$. The source is located at the center of the computational domain with a receiver $2.2 \mathrm{~km}$ far from the source to observe the waveforms. We choose a cubic element mesh of size $55 \mathrm{~m} \times 55 \mathrm{~m} \times 55 \mathrm{~m}$ with $\Delta t=3 \mathrm{~ms}$ for the computation, resulting in about 3.64 elements per minimum wavelength. Fig. 7 gives the snapshots in the $x-y$ plane through the source at $T=1.155 \mathrm{~s}$, as generated by the $P^{2}$ WRKDG method and the 4th-order SG method, respectively. It can be seen that the snapshot generated by the WRKDG method (Fig. 7(a)) is very clear and has no visible numerical dispersion, whereas the 4th-order SG method (Fig. 7(b)) suffers from serious numerical dispersion. To illustrate the problem more precisely, we also show the waveforms at the receiver. Fig. 8 shows the normalized waveforms computed by the $P^{2}$ WRKDG and the 4th-order SG method, compared with the exact solution of the acoustic wave equation computed by the Cagnidard-de Hoop method [33]. From Fig. 8, we can see that the numerical solution
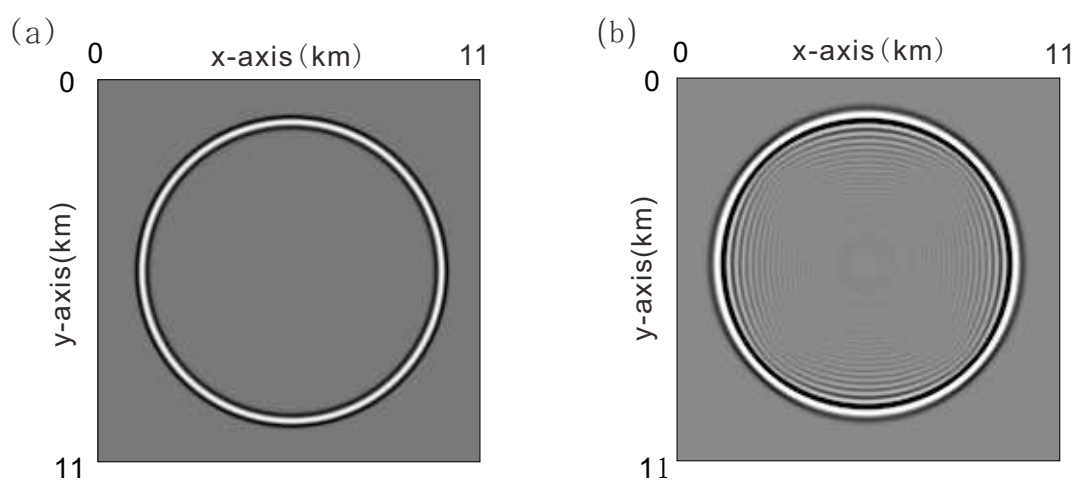

Figure 7: Snapshots of the seismic wave fields at time $T=1.155 \mathrm{~s}$ for homogeneous isotropic acoustic medium using a coarse grid $(55 \mathrm{~m} \times 55 \mathrm{~m} \times 55 \mathrm{~m})$ generated by: (a) WRKDG method for $P^{2}$ element and (b) the 4th-order SG method. 
(a)

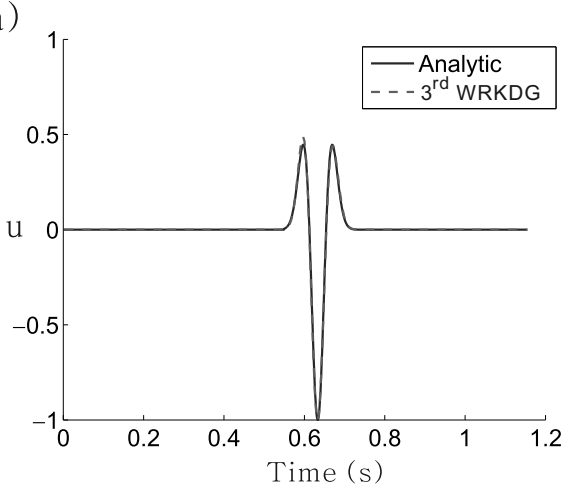

(b)

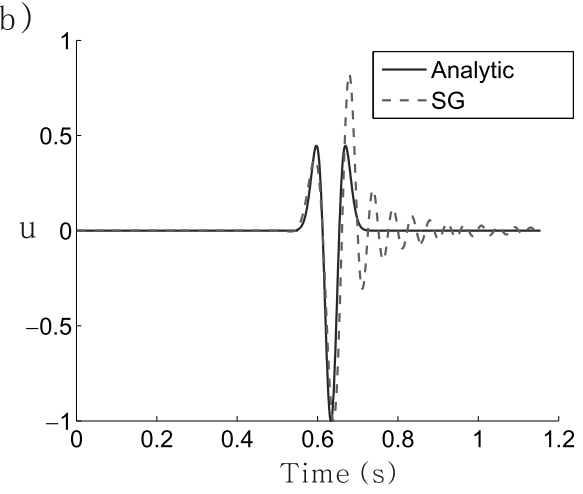

Figure 8: Comparisons of normalized waveforms at time $T=1.155 \mathrm{~s}$ for homogeneous isotropic acoustic medium using a coarse grid $(55 \mathrm{~m} \times 55 \mathrm{~m} \times 55 \mathrm{~m})$ : (a) WRKDG method for $P^{2}$ element versus analytic solution and (b) the 4th-order SG method versus analytic solution.

computed by WRKDG method matches well with the analytic solution, while the waveforms computed by the SG method show some small waves behind the principal wave, which indicates the WRKDG method can suppress numerical dispersion more efficiently than the SG method.

\subsection{Two-layer acoustic model}

In this experiment, we employ the 3D WRKDG method to simulate the wave propagation in the medium with large velocity contrast. The velocities are $2 \mathrm{~km} / \mathrm{s}$ and $4 \mathrm{~km} / \mathrm{s}$ in the upper and lower layers, respectively, which introduces a velocity contrast of 2 times. The computational region is $0 \leq x, y, z \leq 7.2 \mathrm{~km}$, with an interface at $z=3.6 \mathrm{~km}$. The source is located at this point $(3.6 \mathrm{~km}, 3.6 \mathrm{~km}, 2.91 \mathrm{~km})$, and the source function is the same as that used in Eq. (5.2) with a peak frequency of $f_{0}=20 \mathrm{~Hz}$. The mesh size is $30 \mathrm{~m} \times$ $30 \mathrm{~m} \times 30 \mathrm{~m}$ and $\Delta t=1.9 \mathrm{~ms}$ for the computation, resulting in about 3.33 elements per minimum wavelength. Fig. 9 shows the snapshots in the $x-y, x-z$, and $y-z$ plane through the source at $T=1.2 \mathrm{~s}$, as generated by the $P^{2}$ element WRKDG method and 4th-order SG method, respectively. It can be seen that the snapshots generated by the WRKDG method (Figs. 9(a-c)) are very clear and have no visible numerical dispersion, whereas the 4th-order SG method (Figs. 9(d-f)) suffers from serious numerical dispersion, which demonstrates that the 3D WRKDG method behaves well even when there is large velocity contrast between the adjacent layers.

\subsection{Homogeneous isotropic elastic model}

In this example, we use the 3D WRKDG method to simulate the elastic wave propagating in the homogeneous isotropic medium. The Lamé parameters are set to be $\lambda=4.704 \mathrm{GPa}$, $\mu=8.4 \mathrm{GPa}$, and the density is $2100 \mathrm{~kg} / \mathrm{cm}^{3}$, resulting in the $P$-wave velocity $3.2 \mathrm{~km} / \mathrm{s}$ 

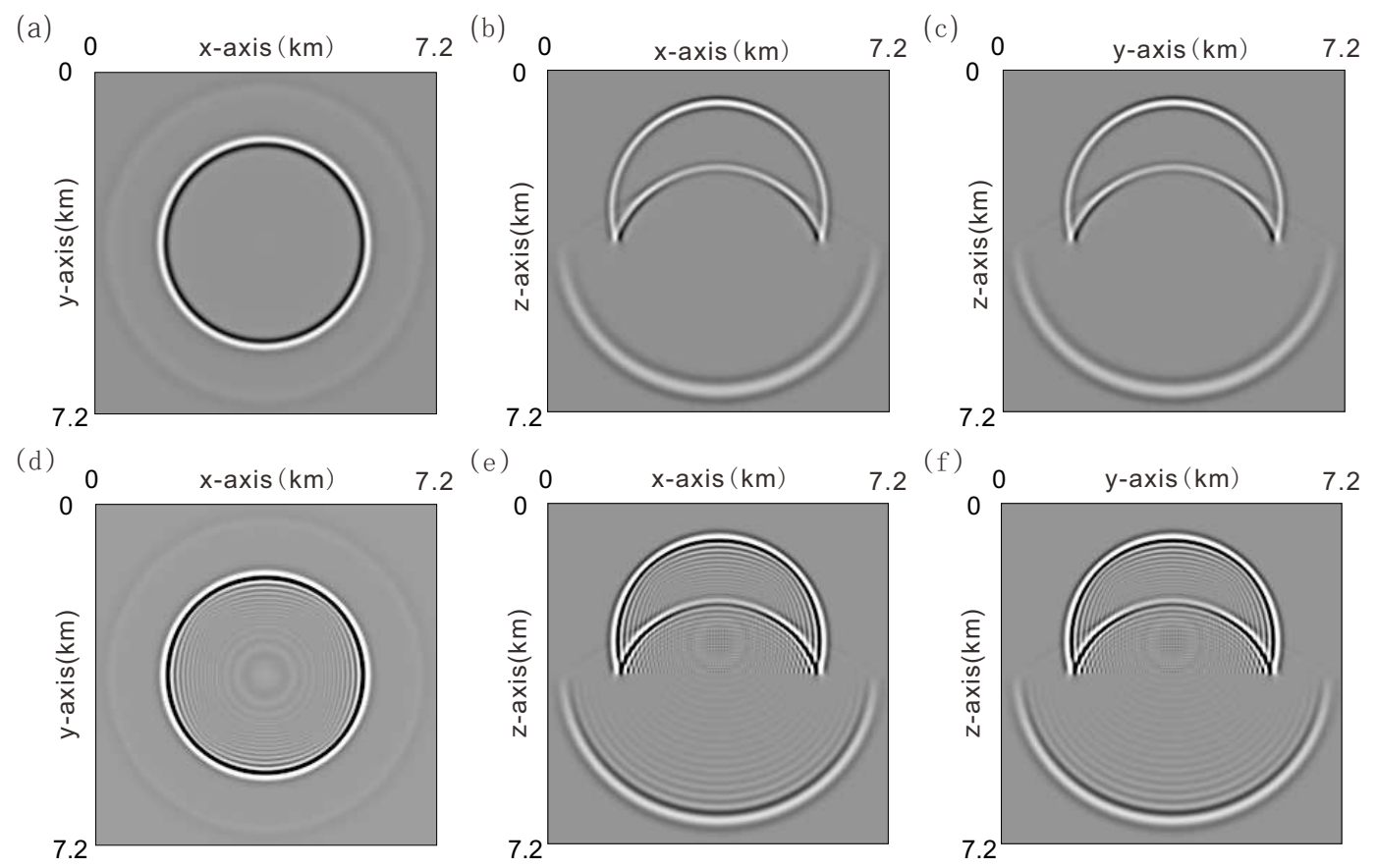

Figure 9: Snapshots for the two-layer acoustic medium in the $x-y$ plane, $x-z$ plane and $y-z$ plane through the source at $T=1.2 \mathrm{~s}$, as generated by (a-c) 3D WRKDG method and (d-f) 4th-order SG method, respectively.

and the $S$-wave velocity $2 \mathrm{~km} / \mathrm{s}$. The computational region is $0 \leq x, y, z \leq 6.0 \mathrm{~km}$. The source is located at the center of the domain, with a body source function:

$$
f_{1}(t)=f_{2}(t)=f_{3}(t)=f(t)
$$

The peak frequency is $20 \mathrm{~Hz}$. The mesh size is $25 \mathrm{~m} \times 25 \mathrm{~m} \times 25 \mathrm{~m}$ with a time step $\Delta t=2$ $\mathrm{ms}$ for the computation, resulting in about 4 elements per minimum wavelength. Fig. 10 shows the snapshots in the $x-y, x-z$, and $y-z$ plane through the source for the displacement vector $\left(u_{1}, u_{2}, u_{3}\right)$ at $T=0.85 \mathrm{~s}$, in which the propagation for the $P$-wave and $S$-wave are clearly observed with no visible numerical dispersion.

\subsection{Two-layer elastic model}

In this example, we use the 3D WRKDG method to simulate the elastic waves propagating in the two-layer medium. The computational region is $0 \leq x, y, z \leq 6.0 \mathrm{~km}$. A horizontal interface is set at the depth of $z=3.75 \mathrm{~km}$. The source is located at $(3 \mathrm{~km}, 3$ $\mathrm{km}, 3.4 \mathrm{~km}$ ) whose body source function is the same as Eq. (5.3) with a peak frequency of $20 \mathrm{~Hz}$. The Lamé parameters, the density, and elastic wave velocities are shown in Table 2 for the two-layer model. In this experiment, we choose the spatial increments of $\Delta \mathrm{x}=\Delta y=\Delta z=20 \mathrm{~m}$ and the time step $\Delta t=1.9 \mathrm{~ms}$. 

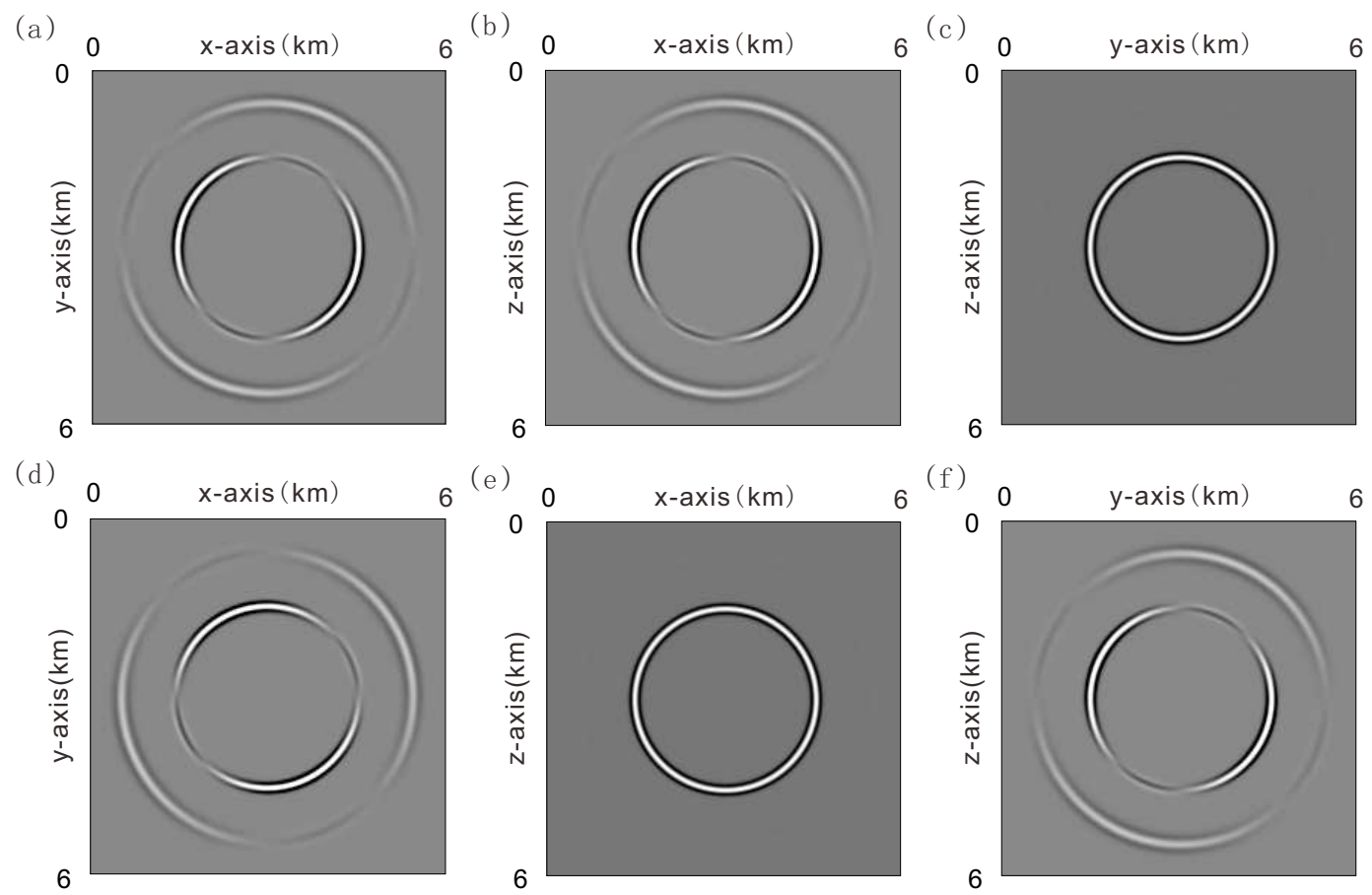

6 (e)
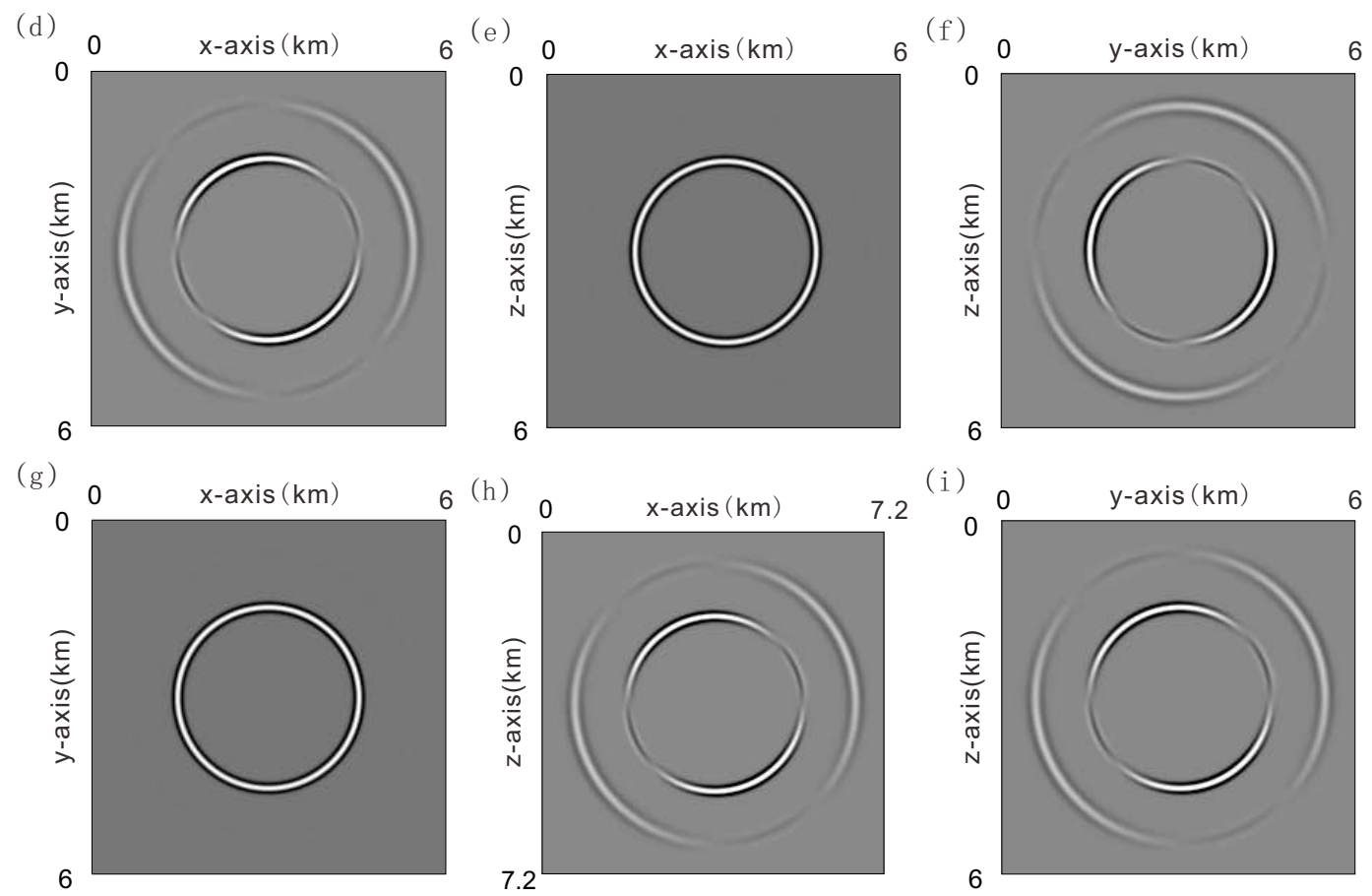

Figure 10: Snapshots for homogeneous isotropic elastic medium in the $x-y$ plane, $x-z$ plane and $y-z$ plane through the source at $T=0.85 \mathrm{~s}$ generated by 3D WRKDG method, in which (a-c), (d-f) and (g-i) are for $u_{1}$, $u_{2}$ and $u_{3}$, respectively.

Table 2: Parameters for two-layer elastic medium.

\begin{tabular}{||l|c|c|c|c|c||}
\hline & $\lambda(\mathrm{GPa})$ & $\mu(\mathrm{GPa})$ & $\rho\left(\mathrm{g} / \mathrm{cm}^{3}\right)$ & $V_{P}(\mathrm{~km} / \mathrm{s})$ & $V_{S}(\mathrm{~km} / \mathrm{s})$ \\
\hline Upper layer & 1.5 & 2.5 & 1.5 & 2.08 & 1.29 \\
\hline Lower layer & 4.5 & 8.5 & 2.1 & 3.20 & 2.01 \\
\hline
\end{tabular}

Fig. 11 shows the wave-field snapshots of displacement vector $\left(u_{1}, u_{2}, u_{3}\right)$ at $T=0.95$ $\mathrm{s}$ in the $x-y, x-z$, and $y-z$ planes through the source. The propagation for the direct $P$ wave and direct $S$-wave are clearly observed. Similarly, the reflected, transmitted, and 

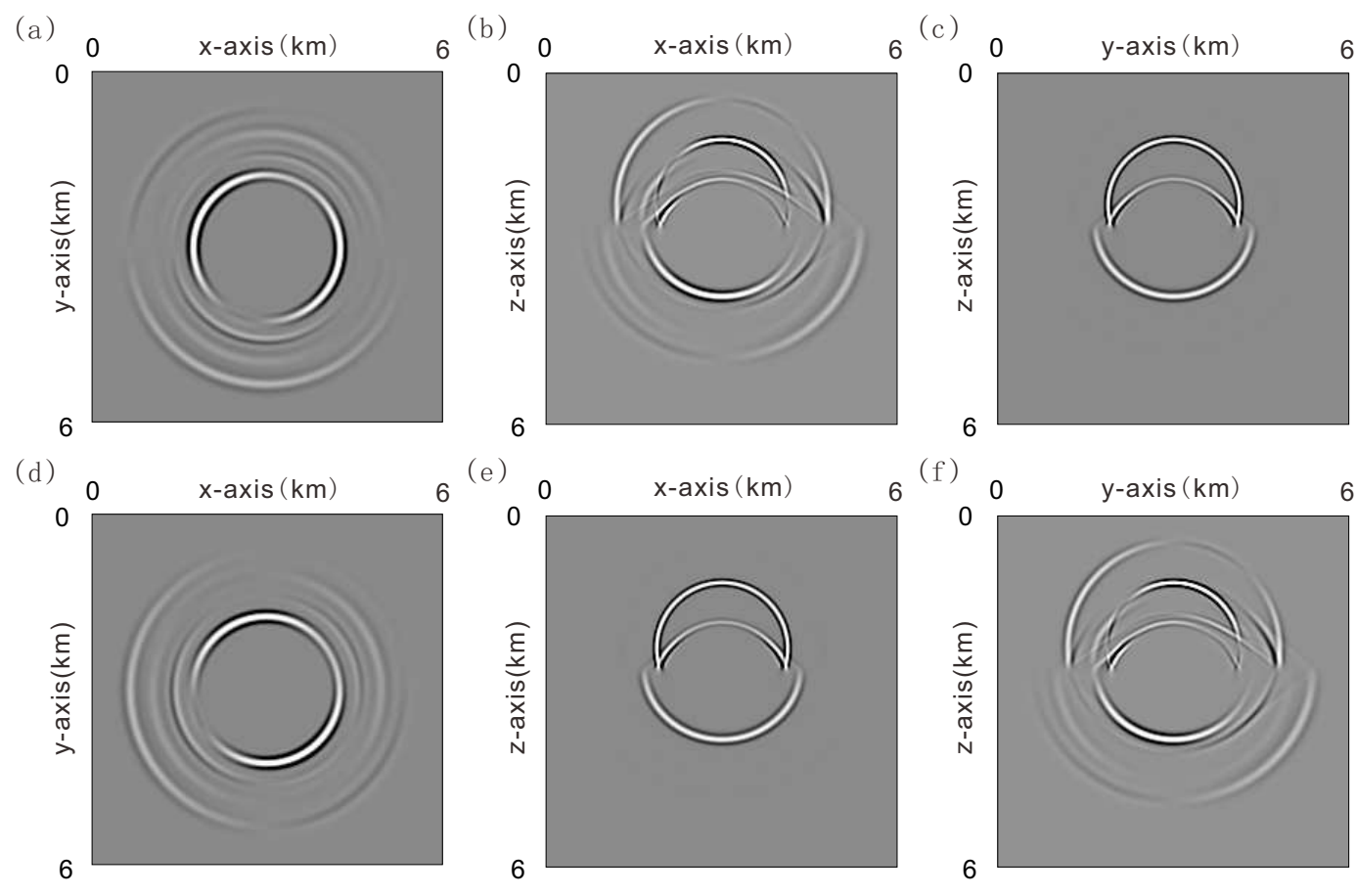

$6 \stackrel{(e)}{0}$

6

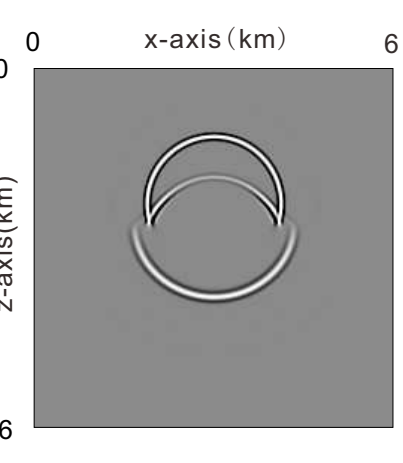

$6^{(f)} 0 \quad y$-axis $(\mathrm{km}) \quad 6$

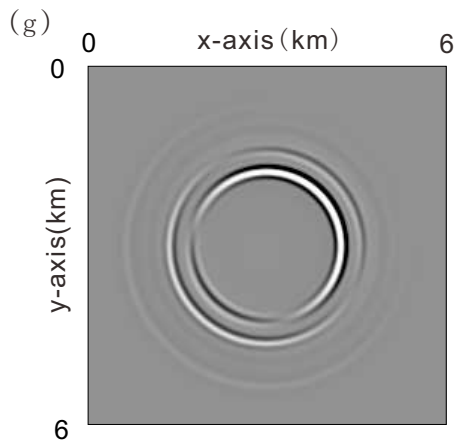

$6^{(h)} 0$

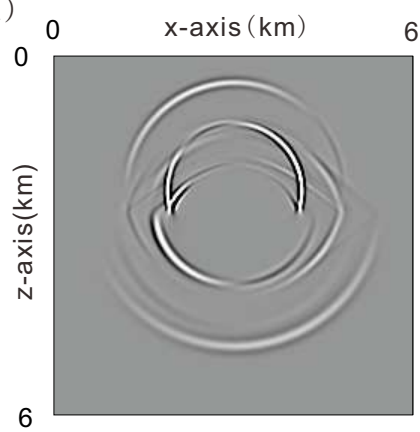

6
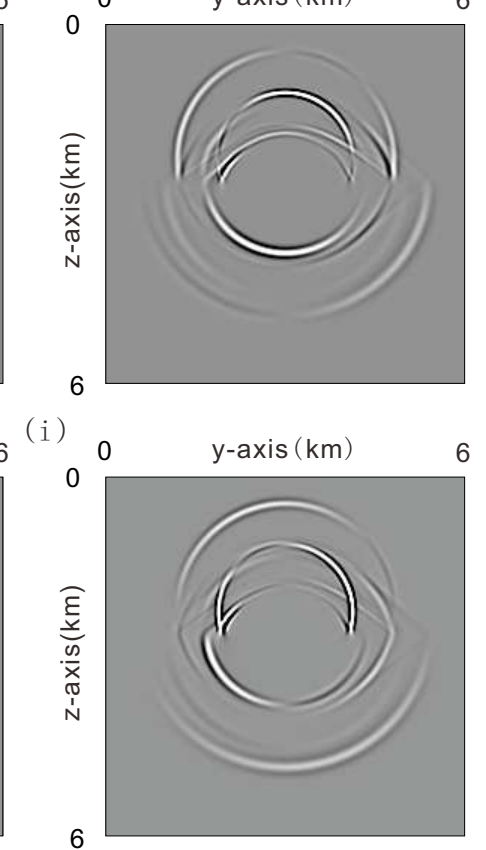

Figure 11: Snapshots for two-layer elastic medium in the $x-y$ plane, $x-z$ plane and $y-z$ plane through the source at $T=0.95 \mathrm{~s}$ generated by 3D WRKDG method, in which (a-c), (d-f) and (g-i) are for $u_{1}, u_{2}$ and $u_{3}$, respectively.

converted waves caused by the interface can be clearly identified from the snapshots by means of the difference in amplitude and different wave phases shown in Fig. 12. The snapshots are clear with no visible numerical dispersion. These results show that the 3D WRKDG method performs well for complicated wave-propagation phenomena.

\subsection{Vertical transversely isotropic (VTI) model}

In the final example, we simulate the elastic wave propagating in the homogeneous VTI medium (Eq. (2.3)). The elastic parameters are set to be $c_{11}=20 \mathrm{GPa}, c_{33}=17.5 \mathrm{GPa}, c_{12}=6$ $\mathrm{GPa}, c_{13}=4.5 \mathrm{GPa}, c_{44}=4 \mathrm{GPa}, \rho=1800 \mathrm{~kg} / \mathrm{cm}^{3}, c_{66}=7 \mathrm{GPa}$. The computational region 


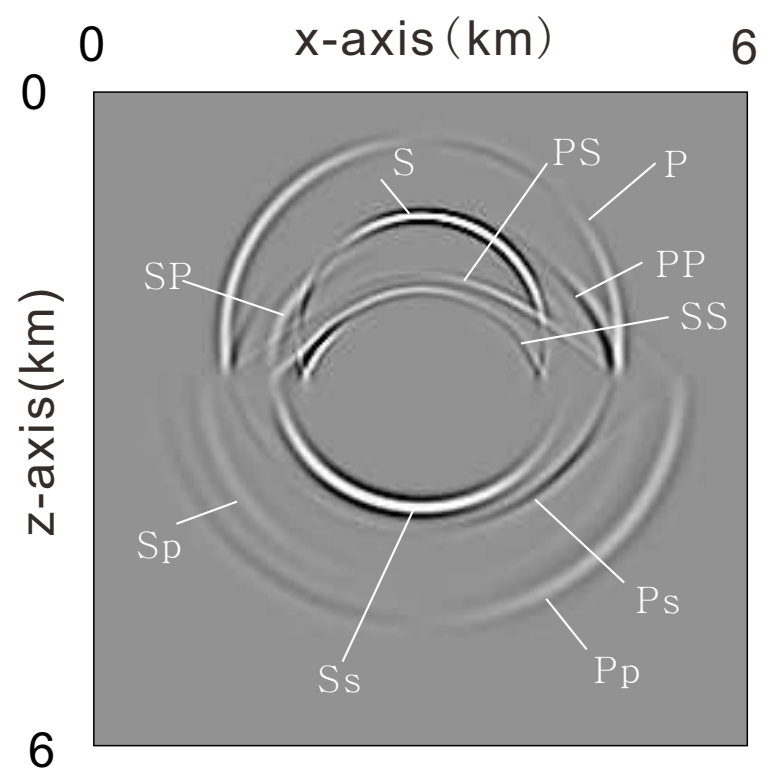

Figure 12: Snapshot for the reflected, transmitted, and converted waves caused by the interface for displacement component $u_{1}$ in the $x-z$ plane (a larger version of Fig. 11(b)). We use the notations for these reflected, transmitted, and converted waves: $P$, direct $P$ wave; $S$, direct $S$ wave; $P P$, reflected $P$ wave; $P S$, reflected $P$-to-converted $S$ wave; $S S$, reflected $S$ wave; $S P$, reflected $S$-to-converted $P$ wave; $P p$, transmitted $P$ wave; $P S$, transmitted $P$-to-converted $S$ wave; $S$ s, transmitted $S$ wave; $S p$, transmitted $S$-to-converted $P$ wave.

is $0 \leq x, y, z \leq 6.0 \mathrm{~km}$. The source is located at the center of the domain whose body source function is the same as Eq. (5.3) with a peak frequency of $20 \mathrm{~Hz}$. In this experiment, we choose the spatial increments of $\Delta \mathrm{x}=\Delta y=\Delta z=25 \mathrm{~m}$ and the time step $\Delta t=2.5 \mathrm{~ms}$.

Fig. 13 shows the wave-field snapshots of the displacement vector $\left(u_{1}, u_{2}, u_{3}\right)$ at $T=$ $0.85 \mathrm{~s}$ in the $x-y, x-z$, and $y-z$ planes through the source. We can observe the anisotropic characteristics for the elastic wave propagation. Since the elastic wave for the VTI model in the $x-y$ plane is isotropic, we observe some concentric circles from Figs. 14(a), 14(d) and $14(\mathrm{~g})$. However, the $P$ - and $S$-waves show anisotropic in $x-z$ and $y-z$ planes, and from the wav-field snapshots we can clearly observe the cusps, triplications and $S$-wave splitting.

\section{Discussion and conclusions}

In this article, we suggest a 3D WRKDG method and apply it for the 3D seismic wave field modeling. The DGM inherits the FEM framework and can deal with curve boundaries by using irregular grids, hence it is suitable for handling surface tomography problems and some special structural such as media with caves and fractures. The word "discontinuous" in DGM implies that we can implement broken basis functions across element interfaces and therefore the method is especially suitable for solving the physical problems with discontinuous characteristics such as shock, complex interfaces between solids and fluids, etc. 

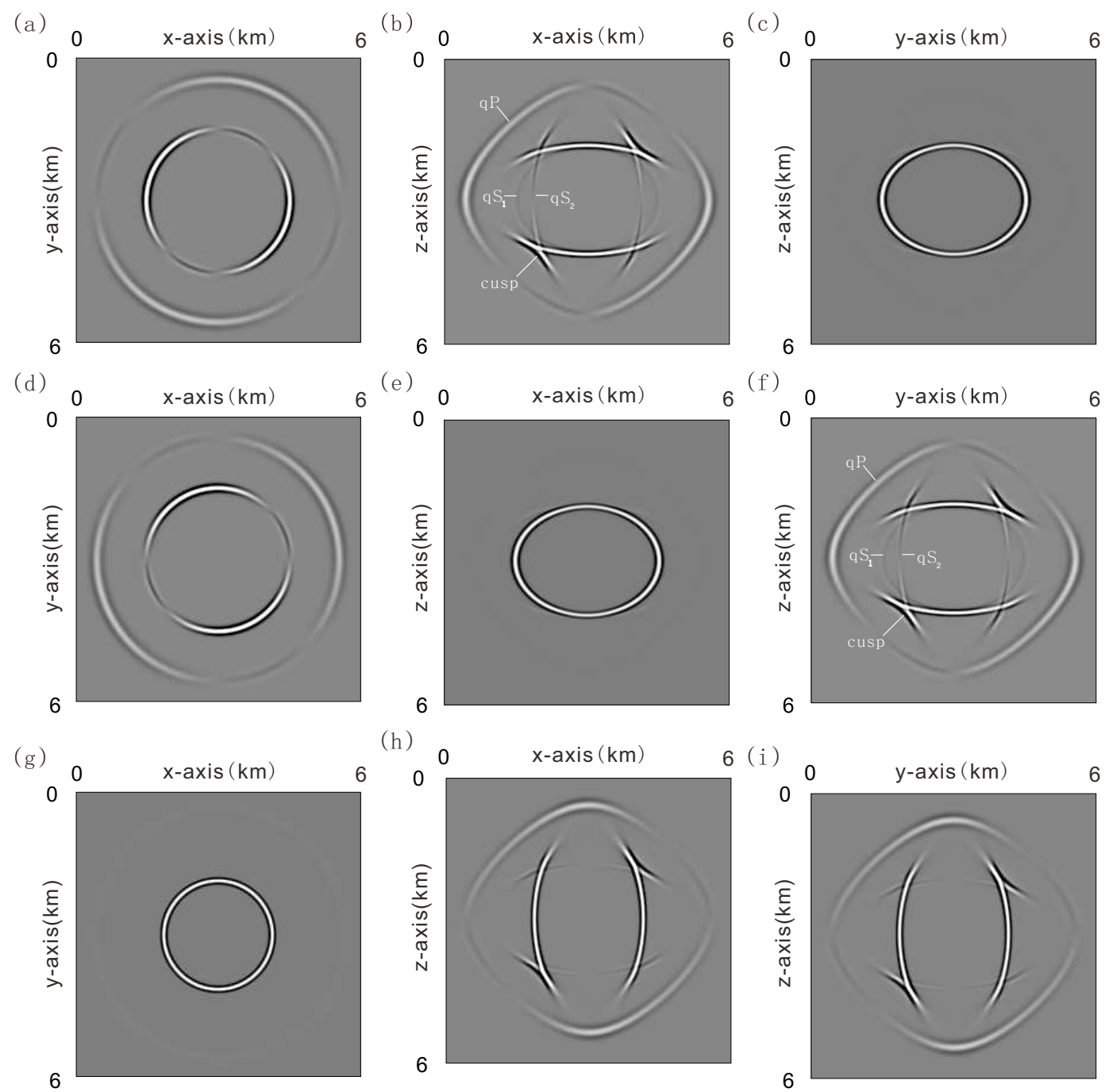

Figure 13: Snapshots for VTI model in the $x-y$ plane, $x-z$ plane and $y-z$ plane through the source at $T=0.85 \mathrm{~s}$ generated by 3D WRKDG method, in which (a-c), (d-f) and (g-i) are for $u_{1}, u_{2}$ and $u_{3}$, respectively.

For the proposed 3D WRKDG method, we employ the numerical flux formulae of DGM, and the time discretization is based on an implicit diagonal RK method, which is then transformed into an explicit method by a two-step iterative process. In addition, the introduction of a weighting factor in the time discretization process enriches the method. The stability conditions of the proposed method are investigated and compared with those of the classic TVD RK DGM. We conclude that the CFL stability conditions of the 3D WRKDG for the acoustic case are more relaxed compared to that of the 3D TVD RK DGM. In particular, the maximal Courant numbers for the $P^{1}$ and $P^{2}$ element methods are 0.888 and 0.310 , respectively, which are roughly 3.4 and 2.3 times larger than that 
of the 3D TVD RK DGM, respectively. Although the computational cost for each timemarching is 2 times larger than the TVD RK time discretization, the weighted RK time stepping scheme still achieve a 1.7 times superiority for $P^{1}$ element and is comparable to the TVD RK time discretization for $P^{2}$ element in computational efficiency.

The numerical dispersion of the 3D WRKDG method is investigated and compared with the conventional 4th-order SG methods. Results of the analyses show that for $S_{p} \in[0,0.5], \theta \in[0, \pi], \varphi \in[0,2 \pi]$, the numerical dispersion error of the $P^{2}$ WRKDG method is no larger than $3.543 \%$, which is much smaller than that of the SG method. Moreover, we observe much smaller dispersive anisotropy for our proposed method. Thereupon the WRKDG method is very suitable for wave simulations since we can reduce both the computational cost and the memory requirement by using a coarser spatial grid increment to achieve the same accuracy as those of other method on a finer grid increment. To further test the capability of the 3D WRKDG scheme to suppress the numerical dispersion, we conduct several typical 3D seismic models, including homogeneous and heterogeneous media, isotropic and anisotropic cases on coarse grids. Two-layer acoustic model and elastic model indicate that 3D WRKDG can provide accurate wave-field without any special treatment at the interfaces. The wave-field snapshots for VTI model clearly show the cusps, triplications and $S$-wave splitting, indicating that the 3D WRKDG method is able to simulate complicated wave propagation.

Based on the above analyses and numerical results for the proposed 3D WRKDG method, we can introduce some deep insights. As we known, when compared to traditional FEMs and other numerical methods, the stencil for DG method is completely compact. The stencil is independent of the space discretization order, and is also independent of complexity of the model. This means that all the calculations in a discretization element are limited only to this element and its immediate neighbors. The compactness of the stencil makes DGM especially suitable for parallelization and local refinement of approximated polynomial degrees and element shapes, which can efficiently reduce the communication burden when employing MPI or graphics processing unit (GPU) parallel programming, and can efficiently provide more accurate information for some local complicated structures. Moreover, in the proposed 3D WRKDG method, we introduce some additional variables to transform the second-order wave equations to a first-order hyperbolic system, hence it is also suitable for the velocity-stress form of the seismic wave equations. The additional variables increase the DOF and calculation burden in each element, but they can effectively reduce the numerical dispersion and be used directly in seismic tomography and migration, avoiding the numerical reconstructions of these information. In our view, the 3D WRKDG can play a significant role in large-scale seismic modeling, reverse time migration, and inversion based on the wave equations, particularly in regard to simulations in complex media.

It should be noted that we have restricted our stability conditions and numerical dispersion analyses for the 3D acoustic case with polynomials of degree one and two spatial interpolations, therefore the conclusions are not applicable for the elastic case and for high-order interpolations. However, the analysis approach is general and can be straight- 
forwardly extended to high-order interpolations and the elastic case. In addition, in this paper the 3D WRKDG is applied to wave propagation simulations using regular hexahedrons, the irregular hexahedral meshes or tetrahedral meshes are not yet applied. We are currently working on the extensions of irregular grids and its parallel programming technology. In conclusion, 3D WRKDG method is a promising and potential method worth continuing research in the future.

\section{Acknowledgments}

This work was supported by the Hainan Provincial Natural Science Foundation of China (Grant No. 418QN205), the National Natural Science Foundation of China (Grant Nos. 41604090, 91730306), the National Key R\&D Program on Monitoring, Early Warning and Prevention of Major Natural Disaster (Grant No. 2017YFC1500301), and by the Statoil Company (Contract No. 4503294711). We would like to thank two anonymous reviewers for their helpful remarks and suggestions that greatly improve the manuscript.

\section{Appendices}

\section{A The matrix expressions in Eq. (4.4)}

Hu et al. [23] gave detailed expressions for the matrices used in Eq. (4.4) for the 2D case. In this paper, we generalize these matrices to the $3 \mathrm{D}$ case. The time-independent polynomial basis functions, denoted as $\left\{w_{l}(x, y, z) \mid l=0,1, \cdots, d_{l o c}-1\right\}$, are defined on the reference element $E=[-1,1] \times[-1,1] \times[-1,1]$. For example, for the Legendre basis functions, $\left\{w_{l}(x, y, z) \mid l=0,1, \cdots, d_{l o c}-1\right\}$ are:

$$
\left\{1, x, y, z, x y, x z, y z, \frac{3}{2} x^{2}-\frac{1}{2}, \frac{3}{2} y^{2}-\frac{1}{2}, \frac{3}{2} z^{2}-\frac{1}{2}, \cdots\right\} .
$$

$d_{l o c}$ is the number of the basis functions in element $E$. Then, the entries of the matrices in Eq. (4.4) are:

$$
\begin{aligned}
\{Q\}_{i j}= & \delta_{\alpha \beta} \int_{-1}^{1} \int_{-1}^{1} \int_{-1}^{1} w_{l^{\prime}} w_{l} d x d y d z, \\
\left\{N_{0}\right\}_{i j}= & \left\{\frac{A_{1}+C_{F_{1}} I}{2}\right\}_{\alpha \beta} \int_{-1}^{1} \int_{-1}^{1} w_{l^{\prime}}(1, y, z) w_{l}(1, y, z) d y d z \\
& -\left\{\frac{-A_{1}+C_{F_{2}} I}{2}\right\}_{\alpha \beta} \int_{-1}^{1} \int_{-1}^{1} w_{l^{\prime}}(-1, y, z) w_{l}(-1, y, z) d y d z \\
& -\left\{A_{1}\right\}_{\alpha \beta} \int_{-1}^{1} \int_{-1}^{1} \int_{-1}^{1} \frac{\partial w_{l^{\prime}}}{\partial x} w_{l} d x d y d z,
\end{aligned}
$$




$$
\begin{aligned}
& \left\{N_{+1}\right\}_{i j}=\left\{\frac{A_{1}-C_{F_{1}} I}{2}\right\}_{\alpha \beta} \int_{-1}^{1} \int_{-1}^{1} w_{l^{\prime}}(1, y, z) w_{l}(-1, y, z) d y d z, \\
& \left\{N_{-1}\right\}_{i j}=\left\{\frac{-A_{1}-C_{F_{2}} I}{2}\right\}_{\alpha \beta} \int_{-1}^{1} \int_{-1}^{1} w_{l^{\prime}}(1, y, z) w_{l}(-1, y, z) d y d z, \\
& \left\{M_{0}\right\}_{i j}=\left\{\frac{A_{2}+C_{F_{3}} I}{2}\right\}_{\alpha \beta} \int_{-1}^{1} \int_{-1}^{1} w_{l^{\prime}}(x, 1, z) w_{l}(x, 1, z) d x d z \\
& -\left\{\frac{-A_{2}+C_{F_{4}} I}{2}\right\}_{\alpha \beta} \int_{-1}^{1} \int_{-1}^{1} w_{l^{\prime}}(x,-1, z) w_{l}(x,-1, z) d x d z \\
& -\left\{A_{2}\right\}_{\alpha \beta} \int_{-1}^{1} \int_{-1}^{1} \int_{-1}^{1} \frac{\partial w_{l^{\prime}}}{\partial y} w_{l} d x d y d z \\
& \left\{M_{+1}\right\}_{i j}=\left\{\frac{A_{2}-C_{F_{3}} I}{2}\right\}_{\alpha \beta} \int_{-1}^{1} \int_{-1}^{1} w_{l^{\prime}}(x, 1, z) w_{l}(x,-1, z) d x d z \text {, } \\
& \left\{M_{-1}\right\}_{i j}=\left\{\frac{-A_{2}-C_{F_{4}} I}{2}\right\}_{\alpha \beta} \int_{-1}^{1} \int_{-1}^{1} w_{l^{\prime}}(x,-1, z) w_{l}(x, 1, z) d x d z \text {, } \\
& \left\{K_{0}\right\}_{i j}=\left\{\frac{A_{3}+C_{F_{5}} I}{2}\right\}_{\alpha \beta} \int_{-1}^{1} \int_{-1}^{1} w_{l^{\prime}}(x, y, 1) w_{l}(x, y, 1) d x d y \\
& -\left\{\frac{-A_{3}+C_{F_{6}} I}{2}\right\}_{\alpha \beta} \int_{-1}^{1} \int_{-1}^{1} w_{l^{\prime}}(x, y,-1) w_{l}(x, y,-1) d x d y \\
& -\left\{A_{3}\right\}_{\alpha \beta} \int_{-1}^{1} \int_{-1}^{1} \int_{-1}^{1} \frac{\partial w_{l^{\prime}}}{\partial z} w_{l} d x d y d z, \\
& \left\{K_{+1}\right\}_{i j}=\left\{\frac{A_{3}-C_{F_{5}} I}{2}\right\}_{\alpha \beta} \int_{-1}^{1} \int_{-1}^{1} w_{l^{\prime}}(x, y, 1) w_{l}(x, y,-1) d x d y \text {, } \\
& \left\{K_{-1}\right\}_{i j}=\left\{\frac{-A_{3}-C_{F_{6}} I}{2}\right\}_{\alpha \beta} \int_{-1}^{1} \int_{-1}^{1} w_{l^{\prime}}(x, y, 1) w_{l}(x, y,-1) d x d y,
\end{aligned}
$$

where

$$
\delta_{\alpha \beta}= \begin{cases}1, & \text { if } \alpha=\beta, \\ 0, & \text { otherwise, }\end{cases}
$$

and $i=m l^{\prime}+\alpha, j=m l+\beta ; \alpha, \beta=0,1, \cdots m-1 ; l^{\prime}, l=0,1, \cdots, d_{l o c}-1$, in which $m$ is the number of the unknown variables in $W$. The other parameters are defined in the subsection on the plane wave analysis.

\section{B Quadrature formula}

Let $D$ be the sub-element we are considering, in which a quadrature rule is defined through a linear combinations of values on internal nodes $\left\{X_{i}\right\}$ and weights $\left\{w_{i}\right\}$, which 
reads:

$$
\int_{D} f \approx \sum_{i} w_{i} f\left(X_{i}\right)
$$

Through some basis variable substitutions, we can transform the integral on $E^{n, m, k}=$ $\left[x_{n}, x_{n+1}\right] \times\left[y_{m}, y_{m+1}\right] \times\left[z_{k}, z_{k+1}\right]$ into an integral on reference element $E=[-1,1] \times[-1,1] \times$ $[-1,1]$ :

$$
\int_{x_{n}}^{x_{n+1}} \int_{y_{m}}^{y_{m+1}} \int_{z_{k}}^{z_{k+1}} g(x, y, z) d x d z=\frac{\Delta x}{2} \frac{\Delta y}{2} \frac{\Delta z}{2} \int_{-1}^{1} \int_{-1}^{1} \int_{-1}^{1} g(\hat{x}, \hat{y}, \hat{z}) d \hat{x} d \hat{y} d \hat{z} .
$$

Since our basis functions are defined by 3D Legendre polynomials which is constructed by the tensor-product of 1D Legendre polynomials, we apply the 1D GaussLegendre quadrature rule in each direction independently for the integral in Eq. (B.1). For example, for the integral in $x$-direction, the Gauss-Legendre quadrature rule reads:

$$
\int_{-1}^{1} g(\hat{x}) d \hat{x} \approx \sum_{i=1}^{q} w_{i} g\left(x_{i}\right)
$$

where $x_{i}$ is the Gaussian-Legendre nodes defined in the interval $[-1,1], q$ is the number of Gaussian-Legendre nodes, and $w_{i}$ is the weight for $x_{i} . q, w_{i}, x_{i}$ are defined in Table 3.

Table 3: Gauss-Legendre and weights for Gauss-Legendre integral.

\begin{tabular}{||c|c|c||}
\hline$q$ & $w_{i}$ & $s_{i}$ \\
\hline 1 & 2.000000000000 & 0.000000000000 \\
\hline 2 & 1.0000000000000 & 0.577350269189 \\
& 1.000000000000 & 0.577350269189 \\
\hline & 0.5555555555555 & -0.774596669241 \\
3 & 0.8888888888888 & 0.000000000000 \\
& 0.5555555555555 & 0.774596669241 \\
\hline$\cdots$ & $\cdots$ & $\cdots$ \\
\hline
\end{tabular}

\section{References}

[1] W. H. Reed, T. R. Hill, Triangular mesh methods for the neutron transport equation, Los Alamos Scientific Laboratory Report. LA-UR-73-479, (1973).

[2] B. Cockburn and C. W. Shu, TVB Runge-Kutta local projection discontinuous Galerkin finite element method for conservation laws II: General framework, Math. Comp., 52 (1989), 411435.

[3] B. Cockburn and C. W. Shu, The Runge-Kutta discontinuous Galerkin method for conservation laws V: Multidimensional systems, J. Comput. Phys., 141 (1998), 199-224.

[4] B. Rivière and M. F. Wheeler, Discontinuous finite element methods for acoustic and elastic wave problems, Contemporary Mathematics, 329 (2003), 271-282. 
[5] V. Etienne, E. Chaljub, J. Virieux and N. Glinsky, An hp-adaptive discontinuous Galerkin finite-element method for 3-D elastic wave modelling, Geophys. J. Int., 183 (2010), 941-962.

[6] M. Käser and M. Dumbser, An arbitrary high-order discontinuous Galerkin method for elastic waves on unstructured meshes - I. The two-dimensional isotropic case with external source terms, Geophysical Journal International, 166 (2006), 855-877.

[7] M. Käser, M. Dumbser, J. De La Puente and H. Igel, An arbitrary high-order Discontinuous Galerkin method for elastic waves on unstructured meshes - III. Viscoelastic attenuation, Geophys. J. Int., 168 (2007), 224-242.

[8] M. Dumbser, M. Käser and E. F. Toro, An arbitrary high-order Discontinuous Galerkin method for elastic waves on unstructured meshes - V. Local time stepping and p-adaptivity, Geophys. J. Int., 171 (2007), 695-717.

[9] J. De La Puente, M. Käser, M. Dumbser and H. Igel, An arbitrary high-order discontinuous Galerkin method for elastic waves on unstructured meshes - IV. Anisotropy, Geophys. J. Int., 169 (2007), 1210-1228.

[10] C. Pelties, M. Käser, V. Hermann and C. E. Castro, Regular versus irregular meshing for complicated models and their effect on synthetic seismograms, Geophysical Journal International, 183 (2010), 1031-1051.

[11] C. Pelties, J. Puente, J. P. Ampuero, G. B, Brietzke and M. Käser, Three-dimensional dynamic rupture simulation with a high-order discontinuous Galerkin method on unstructured tetrahedral meshes, Journal of Geophysical Research: Solid Earth (1978-2012), 117(B2) (2012).

[12] S. Minisini, E. Zhebel, A. Kononov and W. A. Mulder, Local time stepping with the discontinuous Galerkin method for wave propagation in 3D heterogeneous media, Geophysics, 78 (2013), T67-T77.

[13] X. J. He, D. H. Yang and H. Wu, A weighted Runge-Kutta discontinuous Galerkin method for wavefield modelling, Geophysical Journal International, 200 (2015), 1389-1410.

[14] D. H. Yang, X. J. He, X. Ma, Y. J. Zhou and J. S. Li, An optimal nearly analytic discreteweighted Runge-Kutta discontinuous galerkin hybrid method for acoustic wavefield modeling, Geophysics, 81(2016), T251-T263.

[15] P. D. Lax and B. Wendroff, Difference schemes for hyperbolic equations with high order of accuracy. Communications on pure and applied mathematics, 17 (1964), pp.381-398.

[16] E. Hairer, S. P. Nørsett and G. Wanner, Solving Ordinary Differential Equations I: Nonstiff Problems, Springer-Verlag, (1993).

[17] H. Segawa, H. Luo and R. Nourgaliev, A Diagonally Implicit Runge-Kutta Method for the Discontinuous Galerkin Solutions of the Navier-Stokes Equations, In 49th AIAA Aerospace Sciences Meeting including the New Horizons Forum and Aerospace Exposition (p. 685), (2011).

[18] P. Rasetarinera and M. Y. Hussaini, An efficient implicit discontinuous spectral Galerkin method, Journal of Computational Physics 172 (2001): 718-738.

[19] N. C. Nguyen, J. Peraire and B. Cockburn, A hybridizable discontinuous Galerkin method for the incompressible Navier-Stokes equations. In Proceedings of the 48th AIAA Aerospace Sciences Meeting and Exhibit, Orlando, Florida, (2010), 2010-362.

[20] D. H. Yang, N. Wang, S. Chen and G. J. Song, An Explicit Method Based on the Implicit Runge-Kutta Algorithm for Solving Wave Equations, Bull. Seism. Soc. Am., 99 (2009), 33403354.

[21] D. H. Yang, L. Wang, A split-step algorithm with effectively suppressing the numerical dispersion for 3D seismic propagation modeling, Bulletin of the Seismological Society of America., 100 (2010), 1470-1484. 
[22] D. H. Yang, N. Wang, E. Liu, A strong stability-preserving predictor-corrector method for the simulation of elastic wave propagation in anisotropic media, Commun. Comput. Phys., 12 (2012), 1006-1032.

[23] F. Q. Hu, M. Y. Hussaini and P. Rasitarinera, An analysis of the discontinuous Galerkin method for wave propagation problems, J. Comput. Phys., 151 (1999), 921-946.

[24] M. Ainsworth, Dispersive and dissipative behaviour of high order discontinuous Galerkin finite element methods, J. Comput. Phys., 198 (2004), 106-130.

[25] M. Ainsworth, P. Monk and W. Muniz, Dispersive and dissipative properties of discontinuous Galerkin finite element methods for the second-order wave equation, J. Sci. Comput., 27(2006), 5-40.

[26] J. D. De Basabe, M. K. Sen and M. F. Wheeler, The Interior Penalty Discontinuous Galerkin Method for Elastic Wave Propagation: Grid Dispersion, Geophys. J. Int., 175 (2008), 83-93

[27] P. F. Antonietti, C. Marcati, I. Mazzieri and A. Quarteroni, High order discontinuous Galerkin methods on simplicial elements for the elastodynamics equation, Numerical Algorithms, 71 (2016), pp.181-206.

[28] A. Ferroni, P. F. Antonietti, I. Mazzieri and A. Quarteroni, Dispersion-dissipation analysis of 3-D continuous and discontinuous spectral element methods for the elastodynamics equation, Geophysical Journal International, 211 (2016), 1554-1574.

[29] E. F. Toro, Riemann solvers and numerical methods for fluid dynamics, Springer, (1999).

[30] R. J. LeVeque, Finite volume methods for hyperbolic problems, Cambridge university press, (2002).

[31] B. Rivière, Discontinuous Galerkin methods for solving elliptic and parabolic equations: theory and implementation, Society for Industrial and Applied Mathematics, (2008).

[32] P. Moczo, J. Kristek and L. Halada, 3D 4th-order staggered-grid finite-difference schemes: stability and grid dispersion, Bull. Seism. Soc. Am., 90 (2000), 587-603.

[33] K. Aki and P. G. Richards, Quantitative Seismology: Theory and Methods, W.H. Freeman and Co., San Francisco, CA, (1980). 